\title{
Generalized Hesitant Fuzzy Ideals in Semigroups
}

\author{
G. Muhiuddin $\mathbb{D}^{1}{ }^{1}$ A. M. Alanazi, ${ }^{1}$ A. Mahboob, ${ }^{2}$ and D. Al-Kadi ${ }^{3}$ \\ ${ }^{1}$ Department of Mathematics, University of Tabuk, Tabuk 71491, Saudi Arabia \\ ${ }^{2}$ Department of Mathematics, Madanapalle Institute of Technology \& Science, Madanapalle-517325, India \\ ${ }^{3}$ Department of Mathematics and Statistic, College of Science, Taif University, P.O. Box 11099, Taif 21944, Saudi Arabia
}

Correspondence should be addressed to G. Muhiuddin; chishtygm@gmail.com

Received 6 September 2020; Accepted 17 November 2020; Published 22 December 2020

Academic Editor: Hee S. Kim

Copyright (C) 2020 G. Muhiuddin et al. This is an open access article distributed under the Creative Commons Attribution License, which permits unrestricted use, distribution, and reproduction in any medium, provided the original work is properly cited.

\begin{abstract}
In this paper, as a generalization of the concepts of hesitant fuzzy bi-ideals and hesitant fuzzy right (resp. left) ideals of semigroups, the concepts of hesitant fuzzy $(m, n)$-ideals and hesitant fuzzy $(m, 0)$-ideals (resp. $(0, n)$-ideals) are introduced. Furthermore, conditions for a hesitant fuzzy $(m, n)$-ideal $((m, 0)$-ideal, $(0, n)$-ideal) to be a hesitant fuzzy bi-ideal (right ideal, left ideal) are provided. Moreover, several correspondences between bi-ideals (right ideals, left ideals) and hesitant fuzzy ( $m, n)$-ideals $((m, 0)$-ideals, $(0, n)$-ideals $)$ are obtained. Also, the characterizations of different classes of semigroups in terms of their hesitant fuzzy $(m, n)$-ideals and hesitant fuzzy $(m, 0)$-ideals $((0, n)$-ideals $)$ are investigated.
\end{abstract}

\section{Introduction}

The fuzzy set theory introduced by Zadeh has been applied to different fields. Furthermore, in the literature, a number of generalizations and extensions of fuzzy sets have been introduced, for instance, intuitionistic fuzzy sets, interval-valued fuzzy sets, type 2 fuzzy sets, and fuzzy multisets. As a new generalization of fuzzy sets, Torra [1] introduced the notion of hesitant fuzzy sets which permit the membership degree of an element to a set to be represented by a set of possible values between 0 and 1 (see [1, 2]). Torra [1] defined hesitant fuzzy sets in terms of a function that returns a set of membership values for each element in the domain. The hesitant fuzzy set offers a more accurate representation of hesitancy among people in expressing their preferences over objects than the fuzzy set or its classical extensions. This is really helpful to express the hesitancy of people in everyday life. The hesitant fuzzy set is a valuable tool to deal with uncertainty, which can be accurately and ideally described in terms of decision makers' opinions.

Torra [1] defined hesitant fuzzy sets as a function returning a collection of membership values for each domain element. The hesitant fuzzy set offers a more accurate representation of hesitancy among people in expressing their preferences over objects than the fuzzy set or its classical extensions. Fuzzy set theory has been applied to different classes in semigroups (see, for e.g., [3-9]). Also, fuzzy ideal theory of algebraic structures has been studied on various aspects in [10-13].

Hesitant fuzzy set theory was applied to many practical problems, particularly in the field of decision-making (see, for e.g., [1, 2, 14-19]). Later on, Jun and Song applied the notion of hesitant fuzzy sets to MTL-algebras and EQ-algebras (see $[20,21])$. Recently, hesitant fuzzy set theory has been applied to various algebraic structures on different aspects, namely, Jun et al. applied the hesitant fuzzy set theory to BCK/BCI-algebras and semigroups (see [22-25]), and Muhiuddin et al. applied the hesitant fuzzy set theory to residuated lattices, lattice implication algebras, and BCK/BCI-algebras (see [26-35]). Motivated by a lot of work on hesitant fuzzy sets, we introduce the notions of hesitant fuzzy $(m, n)$-ideals, hesitant fuzzy $(m, 0)$-ideals, and hesitant fuzzy $(0, n)$-ideals of a semigroup by generalizing the concept of hesitant fuzzy bi-ideals, hesitant fuzzy right ideals, and hesitant fuzzy left ideals. Furthermore, associated properties of these generalized notions are discussed. Moreover, characterizations of different semigroup classes such as $(m, n)$-regular, $(m, 0)$-regular, and $(0, n)$-regular semigroups in terms of their hesitant fuzzy $(m, n)$-ideals, hesitant fuzzy $(m, 0)$-ideals, and hesitant fuzzy $(0, n)$-ideals are given. 


\section{Preliminaries}

A nonempty set $S$ endowed with an associative binary operation is called a semigroup. Throughout our discussion, $S$ will denote a semigroup unless otherwise mentioned.

A subset $\varnothing \neq \Omega$ of $S$ is called a sub-semigroup of $S$ if $\Omega \Omega \subseteq \Omega$, and $\Omega$ is called the left (resp. right) ideal of $S$ if $S \Omega \subseteq \Omega$ (resp. $\Omega S \subseteq \Omega$ ). If $\Omega$ is both left and right ideals of $S$, then it is called an ideal of $S$. A sub-semigroup $\Omega$ of $S$ is called a bi-ideal of $S$ if $\Omega S \Omega \subseteq \Omega$.

Let $R$ be a reference set. Then, we define the hesitant fuzzy set (HFS) on $R$ in terms of a function $\mathscr{F}^{\mathscr{H}}$ such that when applied to $R$, it returns a subset of $[0,1]$.

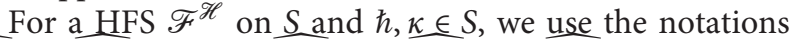
$\widehat{\mathscr{F}}_{\hbar}^{\mathscr{H}}:=\widehat{\mathscr{F}}^{\mathscr{H}}(\hbar)$ and $\mathscr{F}_{\hbar}^{\mathscr{H}} \mathscr{H}:=\widehat{F}^{\mathscr{H}}(\hbar) \cap \widehat{\mathscr{F}}^{\mathscr{H}}(\kappa)$.
Two HFSs $\widehat{\mathscr{F}^{\mathscr{H}}}$ and $\widehat{\mathscr{F}^{\mathscr{H}}} \cap \widehat{\mathscr{G}^{\mathscr{H}}}$ are defined as follows:

$$
\begin{aligned}
& \widehat{\mathscr{F}^{\mathscr{H}}} \cup \widehat{\mathscr{G}^{\mathscr{H}}}: S \longrightarrow \mathscr{\mathscr { P }}([0,1]), \\
& \hbar \mapsto \widehat{\mathscr{F}}_{\hbar}^{\mathscr{H}} \cup \widehat{\mathscr{G}_{\hbar}^{\mathscr{H}}}, \\
& \widehat{\mathscr{F}^{\mathscr{H}}} \cap \widehat{\mathscr{G}^{\mathscr{H}}}: S \longrightarrow\stackrel{\mathscr{P}}{(}[0,1]), \\
& \hbar \mapsto \widehat{\mathscr{F}}_{\hbar}^{\mathscr{H}} \cap \widehat{\mathscr{G}_{\hbar}^{\mathscr{H}}},
\end{aligned}
$$

respectively.

For any HFSs $\widehat{\mathscr{F}^{\mathscr{H}}}$ and $\widehat{\mathscr{G}^{\mathscr{H}}}$ on $S$, we define $\widehat{\mathscr{F}^{\mathscr{H}}} \subseteq \widehat{\mathscr{G}^{\mathscr{H}}}$ if $\widehat{\mathscr{F}_{\hbar}^{\mathscr{H}}} \subseteq \mathscr{G}_{\hbar}^{\mathscr{H}} \forall \hbar \in S$.

For any two HFSs $\widehat{\mathscr{F}^{\mathscr{H}}}$ and $\widehat{\mathscr{G}^{\mathscr{H}}}$ of $S$, the HFS $\widehat{\mathscr{F}^{\mathscr{H}}} \circ \widehat{\mathscr{G}^{\mathscr{H}}}$ is defined as

$$
\left(\widehat{\mathscr{F}^{\mathscr{H}}} \widehat{\mathscr{G}}^{\mathscr{H}}\right)(\hbar)= \begin{cases}\cup_{\hbar=\kappa \ell}\left\{\widehat{\mathscr{F}^{\mathscr{H}}}(\kappa) \cap \widehat{\mathscr{G}^{\mathscr{H}}}(\ell)\right\}, & \text { if there exist } \kappa, \ell \in S \text { such that } \hbar=\kappa \ell, \\ \varnothing, & \text { otherwise. }\end{cases}
$$

For $\Omega \subseteq S$, we denote by $\widehat{\chi_{\Omega}^{\mathscr{H}}}$ the hesitant characteristic fuzzy set of $\Omega$, which is defined as

$$
\widehat{\chi_{\Omega}}(\hbar)= \begin{cases}{[0,1]} & \text { if } \hbar \in \Omega, \\ \varnothing & \text { if } \hbar \notin \Omega .\end{cases}
$$

We denote the identity HFS by $\widehat{\mathscr{F}_{S}^{\mathscr{H}}}$, and it is defined as follows:

$$
\widehat{\mathscr{I}_{S}^{\mathscr{H}}}(\hbar)=[0,1], \quad \forall \hbar \in S .
$$

Let $A, B \subseteq S$. Then, we have

(1) $\widehat{\chi_{A}^{\mathscr{H}}} \widehat{\circ} \widehat{\chi_{\mathrm{B}}}=\widehat{\chi_{\mathrm{AB}}}$.

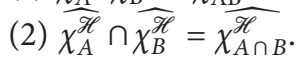

A HFS $\widehat{\mathscr{F}^{\mathscr{H}}}$ is called a hesitant fuzzy sub-semigroup (briefly HFSS) of $S$ if $\forall \hbar, \kappa \in S, \widehat{\mathscr{F}^{\mathscr{H}}}(\hbar \kappa) \supseteq \widehat{\mathscr{F}}^{\mathscr{H}}(\hbar) \cap \widehat{\mathscr{F}^{\mathscr{H}}}(\kappa)$, and $\widehat{\mathscr{F}^{\mathscr{H}}}$ is called a hesitant fuzzy left (resp. right) ideal (briefly HFLI and HFRI) of $S$ if $\forall \hbar, \kappa \in S$, $\widehat{\mathscr{F}^{\mathscr{H}}}(\hbar \kappa) \supseteq \widehat{\mathscr{F}}^{\mathscr{H}}(\kappa)\left(\right.$ resp. $\left.\widehat{\mathscr{F}^{\mathscr{H}}}(\hbar \kappa) \supseteq \widehat{\mathscr{F}}^{\mathscr{H}}(\hbar)\right)$. If $\widehat{\mathscr{F}^{\mathscr{H}}}$ is both HFLI and HFRI of $S$, then it is called a hesitant fuzzy ideal of S. A HFSS $\widehat{\mathscr{F}^{\mathscr{H}}}$ is called a hesitant fuzzy bi-ideal (briefly HFBI) of $S$ if $\widehat{\mathscr{F}^{\mathscr{H}}}(\hbar \ell \kappa) \supseteq \widehat{\mathscr{F}^{\mathscr{H}}}(\hbar) \cap \widehat{\mathscr{F}^{\mathscr{H}}}(\kappa)$ for each $\hbar, \kappa, \ell \in S$.

Throughout the paper, $\widehat{\wp_{\mathscr{H}_{R}}}, \widehat{\wp_{\mathscr{H}_{L}}}$, and $\widehat{\wp_{\mathscr{H}_{B}}}$ will stand for the set of all hesitant fuzzy right ideals, hesitant fuzzy left ideals, and hesitant fuzzy right bi-ideals of $S$.
The concept of $(m, n)$-ideals of semigroups was given by Lajos [36]. Also, the study of $(m, n)$-ideals in different algebraic structures has been conducted by several authors [37-43]. A sub-semigroup $A$ of $S$ is called an $(m, n)$-ideal of $S$ [36] if $A^{m} S A^{n} \subseteq A$, where $m$ and $n$ are nonnegative integers. Here, $A^{0} S=S A^{0}=S$.

The set of all $(m, n)$-ideals, $(m, 0)$-ideals, and $(0, n)$-ideals will be denoted by $\mathscr{I}_{(m, n)}, \mathscr{I}_{(m, 0)}$, and. $\mathscr{I}_{(0, n)}$

\section{Main Results}

Definition 1. A HFSS $\widehat{\mathscr{F}^{\mathscr{H}}}$ of $S$ is called a hesitant fuzzy $(m, n)$-ideal of $S$ if $\widehat{\mathscr{F}}_{r_{1} r_{2} \ldots r_{m} z s_{1} s_{2} \ldots s_{n}}$ $\supseteq \widehat{\mathscr{F}_{r_{1}}^{\mathscr{H}}} \cap \widehat{\mathscr{F}_{r_{2}}^{\mathscr{H}}} \cap \cdots \cap \widehat{\mathscr{F}_{r_{m}}^{\mathscr{H}}} \cap \widehat{\mathscr{F}_{s_{1}}^{\mathscr{H}}} \cap \widehat{\mathscr{F}}_{s_{2}}^{\mathscr{H}} \cap \cdots \cap \widehat{\mathscr{F}}_{s_{n}}^{r_{1} r_{2} \ldots r_{m} z s_{1} s_{2} \ldots s_{n}}$ for all $r_{1}, r_{2}, \ldots, r_{m}, z, s_{1}, s_{2}, \ldots s_{n} \in S$.

Throughout the paper, $\wp \widehat{\mathscr{H}(m, n)}$ will stand for the set of all hesitant fuzzy $(m, n)$-ideals of $S$.

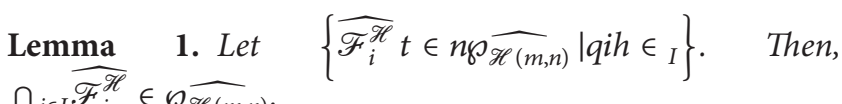
Proof. Straightforward.

Remark 1. Let $\left\{\widehat{\mathscr{F}_{i}^{\mathscr{H}}} t \in n \wp_{\mathscr{\mathscr { H }}(m, n)} q h \mid x i 7 \in C I\right\}$. Then, $\cup_{i \in I} \widehat{\mathscr{F}_{i}^{\mathscr{H}}} \notin \widehat{\wp_{\mathscr{H}(m, n)}}$ in general. We illustrate it by the following example. 
Example 1. Let $S=\{\vartheta, \iota, \kappa, \hbar\}$ be a semigroup with the following multiplication table:

Let $\widetilde{F}_{1}^{\mathscr{H}}$ and $\widetilde{F}_{2}^{\mathscr{H}}$ be two HFS of $S$ such that

$$
\begin{array}{r}
\widehat{\mathscr{F}_{1}^{\mathscr{H}}}(\vartheta)=[0,0.2], \\
\widehat{\mathscr{F}_{1}^{\mathscr{H}}}(\iota)=[0,0.2], \\
\widehat{\mathscr{F}_{1}^{\mathscr{H}}}(\kappa)=\varnothing, \\
\widehat{\mathscr{F}}_{1}^{\mathscr{H}}(\hbar)=\varnothing, \\
\widehat{\mathscr{F}_{2}^{\mathscr{H}}}(\vartheta)=[0,0.2], \\
\widehat{\mathscr{F}_{2}^{\mathscr{H}}}(\iota)=\varnothing, \\
\widehat{\mathscr{F}_{2}^{\mathscr{H}}}(\kappa)=[0,0.2], \\
\widehat{\mathscr{F}^{\mathscr{H}}}(\hbar)=\varnothing .
\end{array}
$$

Then, $\widehat{\mathscr{F}_{1}^{\mathscr{H}}}, \widehat{\mathscr{F}_{2}^{\mathscr{H}}} \in \widehat{\wp_{\mathscr{H}(m, n)}}$ but $\widehat{\mathscr{F}_{1}^{\mathscr{H}}} \cup \widehat{\mathscr{F}_{2}^{\mathscr{H}}} \notin \widehat{\wp_{\mathscr{H}(m, n)}}$ because $\varnothing=\widehat{\mathscr{F}}_{1}^{\mathscr{H}}(u) \cup \widehat{\mathscr{F}_{1}^{\mathscr{H}}}(u)=\left(\widehat{\mathscr{F}}_{1}^{\mathscr{H}} \cup t \widehat{\mathscr{F}}_{2}^{\mathscr{H}}\right)(u)=\left(\widehat{\mathscr{F}}_{1}^{\mathscr{H}} \cup t\right.$ $\left.\widehat{\mathscr{F}_{2}^{\mathscr{H}}}\right)(\mathrm{sc}) \subset\left(\widehat{\mathscr{F}_{1}^{\mathscr{H}}} \cup t \widehat{\mathscr{F}_{2}^{\mathscr{H}}}\right)(s) \cap\left(\widehat{\mathscr{F}}_{1}^{\mathscr{H}} \cup t \widehat{\mathscr{F}}_{2}^{\mathscr{H}}\right)(w)=[0,0.2]$.

Lemma 2. Let $\varnothing \neq A \subseteq S$. Then, $A \in \mathscr{I}_{(m, n)} \Leftrightarrow \widehat{\chi_{A}^{\mathscr{H}}} \in \widehat{\wp_{\mathscr{H}(m, n)}}$. Proof. $\quad(\Rightarrow)$ Let $r_{1}, r_{2}, \ldots, r_{m}, z, s_{1}, s_{2}, \ldots, s_{n} \in S$. Then, the following are observed.

Case 1: if $r_{1} \notin A$ for any $1 \in\{1,2, \ldots, \mathrm{m}\}$, then

$$
\widehat{\chi_{A}^{\mathscr{H}}}\left(r_{1} r_{2} \ldots r_{m} z s_{1} s_{2} \ldots s_{n}\right) \supseteq \widehat{\chi_{A}}\left(r_{1}\right) \cap \widehat{\chi_{A}^{\mathscr{H}}}\left(r_{2}\right) \cap \cdots \cap \widehat{\chi_{A}^{\mathscr{H}}}\left(r_{m}\right) \cap \widehat{\chi_{A}^{\mathscr{H}}}\left(s_{1}\right) \cap \widehat{\chi_{A}^{\mathscr{H}}}\left(s_{2}\right) \cap \cdots \cap \widehat{\chi_{A}}\left(s_{n}\right) .
$$

Case 2: if $s_{\mathrm{J}} \notin A$ for any $\mathrm{J} \in\{1,2, \ldots, n\}$, then

$$
\widehat{\chi_{A}^{\mathscr{H}}}\left(r_{1} r_{2} \ldots r_{m} z s_{1} s_{2} \ldots s_{n}\right) \supseteq \widehat{\chi_{A}}\left(r_{1}\right) \cap \widehat{\chi_{A}}\left(r_{2}\right) \cap \cdots \cap \widehat{\chi_{A}^{\mathscr{H}}}\left(r_{m}\right) \cap \widehat{\chi_{A}}\left(s_{1}\right) \cap \widehat{\chi_{A}^{\mathscr{H}}}\left(s_{2}\right) \cap \cdots \cap \widehat{\chi_{A}}\left(s_{n}\right) .
$$

Case 3: if $r_{1} \notin A$ and $s_{\mathrm{J}} \notin A \forall 1 \in\{1,2, \ldots, \mathrm{m}\}$,

$\mathrm{J} \in\{1,2, \ldots, n\}$, then

$$
\widehat{\chi_{A}^{\mathscr{H}}}\left(r_{1} r_{2} \ldots r_{m} z s_{1} s_{2} \ldots s_{n}\right) \supseteq \widehat{\chi_{A}^{\mathscr{H}}}\left(r_{1}\right) \cap \widehat{\chi_{A}^{\mathscr{H}}}\left(r_{2}\right) \cap \cdots \cap \widehat{\chi_{A}^{\mathscr{H}}}\left(r_{m}\right) \cap \widehat{\chi_{A}^{\mathscr{H}}}\left(s_{1}\right) \cap \widehat{\chi_{A}^{\mathscr{H}}}\left(s_{2}\right) \cap \cdots \cap \widehat{\chi_{A}}\left(s_{n}\right) .
$$

Case 4: if $r_{1}, s_{\mathrm{J}} \in A \forall 1 \in\{1,2, \ldots, \mathrm{m}\}, \mathrm{J} \in\{1,2, \ldots, \mathrm{n}\}$, then $r_{1} r_{2} \ldots r_{m} z s_{1} s_{2} \ldots s_{n} \in A^{m} S A^{n} \subseteq A$. Therefore,

$$
\widehat{\chi_{A}^{\mathscr{H}}}\left(r_{1} r_{2} \ldots r_{m} z s_{1} s_{2} \ldots s_{n}\right)=[0,1] \supseteq \chi_{A}^{\widehat{\mathscr{H}}}\left(r_{1}\right) \cap \widehat{\chi_{A}^{\mathscr{H}}}\left(r_{2}\right) \cap \cdots \cap \widehat{\chi_{A}^{\mathscr{H}}}\left(r_{m}\right) \cap \widehat{\chi_{A}^{\mathscr{H}}}\left(s_{1}\right) \cap \widehat{\chi_{A}^{\mathscr{H}}}\left(s_{2}\right) \cap \cdots \cap \widehat{\chi_{A}}\left(s_{n}\right) .
$$

Hence, $\widehat{\chi_{A}^{\mathscr{H}}} \in \widehat{\wp} \widehat{\mathscr{H}(m, n)}$.

$$
\begin{array}{llr}
(\Leftarrow) \quad \text { Let } \quad x, z, y \in S . \quad \text { If } \quad x, y \in A, & \text { then } \\
\widehat{\chi_{A}^{\mathscr{H}}}\left(x^{m} z y^{n}\right) \supseteq \widehat{\chi_{A}}(x) \cap \widehat{\chi_{A}^{\mathscr{H}}}(y)=[0,1] \quad \text { implies }
\end{array}
$$




\begin{tabular}{lllll}
\hline & $\vartheta$ & $\iota$ & $\kappa$ & \\
\hline$\vartheta$ & $\vartheta$ & $\vartheta$ & $\vartheta$ & \\
$\iota$ & $\vartheta$ & $\vartheta$ & $\hbar$ & \\
$\kappa$ & $\vartheta$ & $\vartheta$ & $\vartheta$ & $\vartheta$ \\
$\hbar$ & $\vartheta$ & $\vartheta$ & $\vartheta$ & $\vartheta$ \\
\hline
\end{tabular}

$\widehat{\chi_{A}^{\mathscr{H}}}\left(x^{m} z y^{n}\right)=[0,1] . \quad$ Therefore, $\quad x^{m} z y^{n} \in A$. Thus, $A^{m} S A^{n} \subseteq A$.

Definition 2. For any HFS $\widehat{\mathscr{F}^{\mathscr{H}}}$ of $S$, the set

$$
\widehat{\mathscr{F}_{T}^{\mathscr{H}}}=\left\{x \in S \mid t \widehat{\mathscr{F}}_{x}^{\mathscr{H}} n \supseteq q T\right\}
$$

where $T \in \mathscr{P}([0,1])$, is said to be a hesitant $T$-level subset of $\mathscr{F}^{\mathscr{H}}$.

Theorem 1. Let $\widehat{\mathscr{F}^{\mathscr{H}}}$ be the HFS of S. Then, the hesitant T-level subset $\widehat{\mathscr{F}}_{T}^{\mathscr{H}} \in \mathscr{I}_{(m, n)} \forall T \in \mathscr{P}([0,1])$, provided $\widehat{\mathscr{F}_{T}^{\mathscr{H}}} \neq \varnothing \Leftrightarrow \widehat{\mathscr{F}^{\mathscr{H}}} \in \wp_{\mathscr{\mathscr { H }}(m, n)}$.

Proof. $(\Rightarrow)$ For any $r_{1}, r_{2}, \ldots, r_{m}, s_{1}, s_{2}, \ldots, s_{n} \in S$ and suppose, to the contrary, that $\widehat{\mathscr{F}}_{{ }_{1}} \cap \widehat{\mathscr{F}^{\mathscr{H}}}{ }_{r_{2}} \cap \ldots \quad \cap \widehat{\mathscr{F}}_{r_{m}}^{\mathscr{H}} \cap \widehat{\mathscr{F}}_{s_{1}}^{\mathscr{H}} \cap$

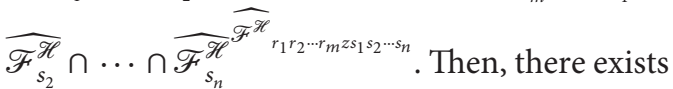
$C \in \mathscr{P}([0,1])$ such that $\widehat{\mathscr{F}_{r_{1}}^{\mathscr{H}}} \cap \widehat{\mathscr{F}_{r_{2}}^{\mathscr{H}}} \cap \cdots \cap \widehat{\mathscr{F}}_{r_{m}}^{\mathscr{H}}$ $\cap \widehat{\mathscr{F}_{s_{1}}^{\mathscr{H}}} \cap \widehat{\mathscr{F}_{s_{2}}^{\mathscr{H}}} \cap \cdots \cap \widehat{\mathscr{F}_{s_{n}}^{\mathscr{H}}} \supseteq C^{\widehat{\mathscr{F}}{ }_{r_{1} r_{2} \cdots r_{m}} z s_{1} s_{2} \cdots s_{n}}$. So, $r_{1}, r_{2}, \ldots, r_{m}, z, s_{1}, s_{2}, \ldots, s_{n} \in \widehat{\mathscr{F}}_{C}^{\mathscr{H}}, \quad$ but $r_{1} r_{2} \cdots r_{m} z s_{1} s_{2} \cdots s_{n} \notin \widehat{\mathscr{F}}_{C}^{\mathscr{H}}, \quad$ a contradiction. Thus, $\quad \widehat{\mathscr{F}}_{r_{1} r_{2} \cdots r_{m} z s_{1} s_{2} \cdots s_{n}} \supseteq \widehat{\mathscr{F}}_{r_{1}}^{\mathscr{H}} \cap$

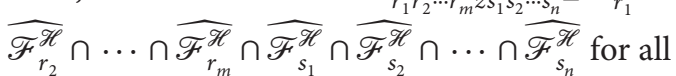
$r_{1}, r_{2}, \ldots, r_{m}, z, s_{1}, s_{2}, \ldots, s_{n} \in S$. Hence, $\widehat{\mathscr{F}^{\mathscr{H}}} \in \widehat{\wp \mathscr{H}(m, n)}$.

$(\Leftarrow)$ Let $z \in S$ and $r_{1}, r_{2}, \ldots, r_{m}, s_{1}, s_{2}, \ldots, s_{n} \in \widehat{\mathscr{F}}_{T}^{\mathscr{H}}$, where $T \in \mathscr{P}([0,1])$. Then, $\widehat{\mathscr{F}}_{r_{1}}^{\mathscr{H}} \supseteq T, \widehat{\mathscr{F}}_{r_{2}}^{\mathscr{H}} \supseteq T, \ldots$, $\widehat{\mathscr{F}_{x_{n}}^{\mathscr{H}}} \supseteq T$ and $\widehat{\mathscr{F}_{s_{1}}^{\mathscr{H}}} \supseteq T, \widehat{\mathscr{F}_{s_{2}}^{\mathscr{H}}} \supseteq T, \cdots \widehat{\mathscr{F}_{y_{m}}^{\mathscr{H}}} \supseteq T$. By definition, $\widehat{\mathscr{F}}_{r_{1} r_{2} \cdots r_{m} z s_{1} s_{2} \cdots s_{n}} \supseteq{\widehat{\mathscr{F}} r_{1}}_{r_{1}} \cap \widehat{\mathscr{F}_{r_{2}}^{\mathscr{H}}} \cap \cdots \cap \widehat{\mathscr{F}_{r_{m}}} \quad{ }_{\mathscr{H}} \cap \widehat{\mathscr{F}_{s_{1}}} \cap$ $\widehat{\mathscr{F}_{s_{2}}^{\mathscr{H}}} \cap \cdots \cap \widehat{\mathscr{F}_{s_{n}}^{\mathscr{H}}}=T$. Therefore, $\quad r_{1} r_{2} \cdots r_{m} z s_{1} s_{2} \cdots$ $s_{n} \in \widehat{\mathscr{F}}_{T}^{\mathscr{H}}$. Hence, $\widehat{\mathscr{F}}_{T}^{\mathscr{H}} \in \mathscr{I}_{(m, n)}$.
Theorem 2. Let $\widehat{\mathscr{F}^{\mathscr{H}}}$ be the HFSS of $S$. Then, $\widehat{\mathscr{F}^{\mathscr{H}}} \in \widehat{\wp}_{\mathscr{H}(m, n)}^{\Leftrightarrow} \widehat{\mathscr{F}}^{m}{\widehat{\mathscr{F}_{S}^{\mathscr{H}}}}^{\circ} \widehat{\mathscr{F}}^{n} \subseteq \widehat{\mathscr{F}}^{\mathscr{H}}$.

Proof. $\quad \Rightarrow \quad$ Let $\quad \widehat{\mathscr{F}^{\mathscr{H}}} \in \widehat{\wp_{\mathscr{H}(m, n)}}$ and $\hbar \in S$. If $\left(\widehat{\mathscr{F}}^{m} \circ \widehat{\mathscr{I}} \mathscr{S}^{\mathscr{H}} \widehat{\mathscr{F}}^{\mathscr{H}}\right)(\hbar)=\varnothing$, then $\widehat{\mathscr{F}}^{\mathrm{H}} \circ \widehat{\mathscr{I}}_{\mathrm{S}}^{\mathscr{H}}$

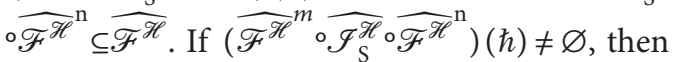
there exist $\ell, \kappa$ in $S$ such that $\hbar=\ell \kappa$, $\left(\widehat{\mathscr{F}}^{m} \circ \widehat{\mathscr{I}}_{S}^{\mathscr{H}}\right)(\ell) \neq \varnothing$, and $\widehat{\mathscr{F}}^{n}(\kappa) \neq \varnothing$. We have the following.

Case 1: when $\left(\widehat{\mathscr{F}}^{\mathscr{H}} \circ{\widehat{\mathscr{J}_{S}^{\mathscr{H}}}}^{\mathscr{P}}\right)(\ell) \neq \varnothing$, then

$\exists u_{1}, v_{1} \in S$ such that $\ell=u_{1} v_{1}$ implies $\widehat{\mathscr{F}}^{m}\left(u_{1}\right) \neq \varnothing$ and $\widehat{\mathscr{I}_{S}^{\mathscr{H}}}\left(v_{1}\right)=[0,1]$

$\exists u_{2}, v_{2} \in S$ such that $u_{1}=u_{2} v_{2} \widehat{\mathscr{F}^{\mathscr{H}}}\left(u_{2}\right) \neq \varnothing$ and $\widehat{\mathscr{F}}^{m-1}\left(v_{2}\right) \neq \varnothing$

$\exists u_{3}, v_{3} \in S$ such that $v_{2}=u_{3} v_{3} \widehat{\mathscr{F}^{\mathscr{H}}}\left(u_{3}\right) \neq \varnothing$ and $\widehat{\mathscr{F}}^{m-2}\left(v_{3}\right) \neq \varnothing$

$\vdots$

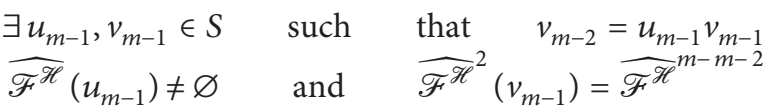
$\left(v_{m-1}\right) \neq \varnothing$

$\exists u_{m}, v_{m} \in S$ such that $v_{m-1}=u_{m} v_{m} \widehat{\mathscr{F}^{\mathscr{H}}}\left(u_{m}\right) \neq \varnothing$ and $\widehat{\mathscr{F}^{\mathscr{H}}}\left(v_{m}\right) \neq \varnothing$

Case 2: when $\widehat{\mathscr{F}}^{n}(\kappa) \neq \varnothing$, then

$\exists u_{1}^{\prime}, v_{1}^{\prime} \in S$ such that $\kappa=u_{1}^{\prime} v_{1}^{\prime} \widehat{\mathscr{F}^{\mathscr{H}}}\left(u_{1}^{\prime}\right) \neq \varnothing \quad$ and $\widehat{\mathscr{F}}^{n-1}\left(v_{1}^{\prime}\right) \neq \varnothing$

$\exists u_{2}^{\prime}, v_{2}^{\prime} \in S$ such that $v_{1}^{\prime}=u_{2}^{\prime} v_{2}^{\prime} \widehat{\mathscr{F}^{\mathscr{H}}}\left(u_{2}^{\prime}\right) \neq \varnothing \quad$ and ${\widehat{\mathscr{F}^{\mathscr{H}}}}^{n-2}\left(v_{2}^{\prime}\right) \neq \varnothing$

$\exists u_{3}^{\prime}, v_{3}^{\prime} \in S$ such that $v_{2}^{\prime}=u_{3}^{\prime} v_{3}^{\prime} \widehat{\mathscr{F}^{\mathscr{H}}}\left(u_{3}^{\prime}\right) \neq \varnothing \quad$ and $\widehat{\mathscr{F}}^{n-3}\left(v_{3}^{\prime}\right) \neq \varnothing$

:

$\exists u_{n-2}{ }^{\prime}, v_{n-2}{ }^{\prime} \in S$ such that $v_{n-3}{ }^{\prime}=u_{n-2}{ }^{\prime} v_{n-2}, \widehat{\mathscr{F}^{\mathscr{H}}}\left(u_{n-2}^{\prime}\right) \neq \varnothing$ and $\widehat{\mathscr{F}}^{\mathscr{H}^{n-(n-2)}}\left(v_{n-2}^{\prime}\right) \neq \varnothing$ 
$\exists u_{n-1}{ }^{\prime}, v_{n-1}{ }^{\prime} \in S$ such that $v_{n-2}{ }^{\prime}=u_{n-1}{ }^{\prime} v_{n-1}{ }^{\prime} \widehat{\mathscr{F}^{\mathscr{H}}}\left(u_{n-1}{ }^{\prime}\right) \neq \varnothing$ and $\widehat{\mathscr{F}^{\mathscr{H}}}\left(v_{n-1}^{\prime}\right) \neq \varnothing$

Now, we have

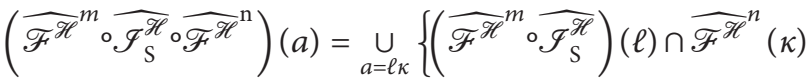

$$
\begin{aligned}
& =\cup_{a=\ell \kappa}\left\{\underset{\ell=u_{1} v_{1}}{\cup}\left\{\widehat{\mathscr{F}}^{m}\left(u_{1}\right) \cap{\widehat{\mathscr{F}_{S}^{\mathscr{H}}}}^{m}\left(v_{1}\right) \cap \underset{\kappa=u_{1}^{\prime} v_{1}^{\prime}}{\cup}\left\{\widehat{\mathscr{F}}^{\mathscr{H}^{\prime}}\left(u_{1}^{\prime}\right) \cap \widehat{\mathscr{F}}^{n-1}\left(v_{1}^{\prime}\right)\right\}\right\}=\right. \\
& u_{a=\ell \kappa} \cup \underset{\ell=u_{1} v_{1}}{\cup} \cup\left\{\widehat{\mathscr{F}}_{\kappa=u_{1}^{\prime} v_{1}^{\prime}}^{m}\left(u_{1}\right) \cap \widehat{\mathscr{F}_{S}^{\mathscr{H}}}\left(v_{1}\right) \cap \widehat{\mathscr{F}^{\mathscr{H}}}\left(u_{1}^{\prime}\right) \cap \widehat{\mathscr{F}}^{n-1}\left(v_{1}^{\prime}\right)\right\}
\end{aligned}
$$

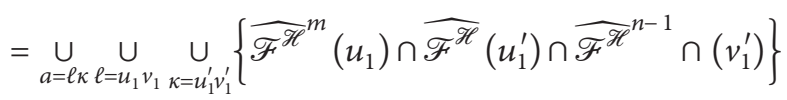

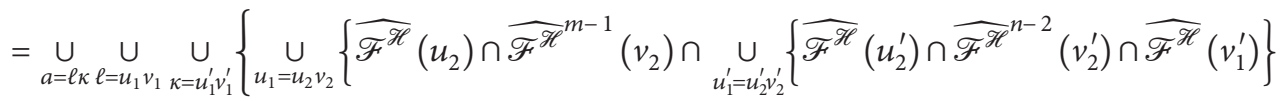

$$
\begin{aligned}
& =\cup_{a=\ell \kappa} \in=u_{1} v_{1} \cup_{\kappa=u_{1}^{\prime} v_{1}^{\prime} u_{1}=u_{2} v_{2} u_{1}^{\prime}=u_{2}^{\prime} v_{2}^{\prime}}\left\{\widehat{\mathscr{F}^{\mathscr{H}}}\left(u_{2}\right) \cap \widehat{\mathscr{F}}^{m-1}\left(v_{2}\right) \cap \widehat{\mathscr{F}^{\mathscr{H}}}\left(u_{2}^{\prime}\right) \cap \widehat{\mathscr{F}}^{n-2}\left(v_{2}^{\prime}\right) \cap \widehat{\mathscr{F}^{\mathscr{H}}}\left(v_{1}^{\prime}\right)\right\}
\end{aligned}
$$

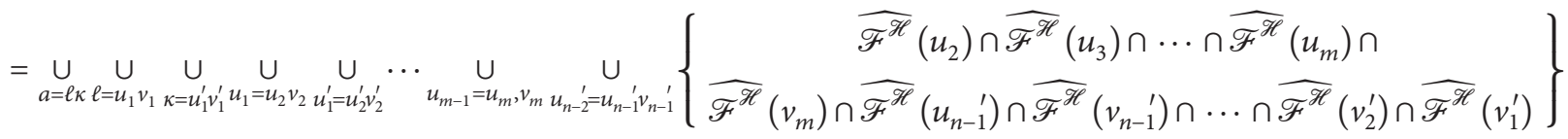

$$
\begin{aligned}
& \subseteq \cup \underset{a=\ell_{\kappa}}{ }\left\{\widehat{\mathscr{F}^{\mathscr{H}}}{ }_{u_{2} u_{3} \ldots v_{m} v_{m} v_{1} u_{1}^{\prime} u_{2}^{\prime} \ldots u_{n-1}^{\prime} v_{n-1}^{\prime}}\right\}=\cup_{a=\ell \kappa}\left\{\widehat{\mathscr{F}^{\mathscr{H}}}(\ell \kappa)\right\} \\
& \text { (since } \left.\ell=u_{2} u_{3} \ldots v_{m} v_{m} v_{1} \text { and } \kappa=u_{1}^{\prime} u_{2}^{\prime} \ldots u_{n-1}^{\prime} v_{n-1}^{\prime}\right)=\widehat{\mathscr{F}^{\mathscr{H}}}(a) \text {. }
\end{aligned}
$$

$(\Leftarrow)$ Assume that ${\widehat{\mathscr{F}^{\mathscr{H}}}}^{m} \circ{\widehat{\mathscr{F}_{\mathrm{S}}}}_{\circ}{\widehat{\mathscr{F}^{\mathscr{H}}}}^{\mathrm{n}} \subseteq \widehat{\mathscr{F}}^{\mathscr{H}}$. For any $r_{1}, r_{2}, \ldots, r_{m}, z, s_{1}, s_{2}, \ldots, s_{n} \in S$,
$a=r_{1} r_{2} \cdots r_{m} z s_{1} s_{2} \cdots s_{n}$. Since $\widehat{\mathscr{F}}^{m} \circ \widehat{\mathscr{F}}_{\mathrm{S}}^{\mathscr{H}} \widehat{\mathscr{F}}^{\mathscr{H}} \subseteq \frac{\text { let }}{\subseteq \mathscr{F}^{\mathscr{H}}}$, we have

$$
\begin{aligned}
& \widehat{\mathscr{F}^{\mathscr{H}}}\left(r_{1} r_{2} \ldots r_{m} z s_{1} s_{2} \ldots s_{n}\right)=\widehat{\mathscr{F}^{\mathscr{H}}}(a) \supseteq\left(\widehat{\mathscr{F}}^{m} \circ{\widehat{\mathscr{F}_{S}^{\mathscr{H}}}}^{\circ \mathscr{F}^{\mathscr{H}}} \mathrm{n}\right)(a) \\
& =\cup_{a=p q}\left\{( \widehat { \mathscr { F } } ^ { m } \circ { \widehat { \mathscr { F } _ { S } ^ { \mathscr { H } } } } ^ { \prime } ( p ) \cap \widehat { \mathscr { F } } ^ { n } ( q ) \} \supseteq \left\{\left(\widehat{\mathscr{F}}^{m} \widehat{\circ}_{\mathscr{F}_{\mathrm{S}}^{\mathscr{H}}}\right)\left(r_{1} r_{2} \ldots r_{m} z\right) \cap \widehat{\mathscr{F}}^{n}\left(s_{1} s_{2} \ldots s_{n}\right)\right.\right. \\
& \supseteq\left\{\underset{r_{1} r_{2} \ldots r_{m} z=u v}{U}\left\{\widehat{\mathscr{F}}^{m}(u) \cap \widehat{\mathscr{F}_{S}^{\mathscr{H}}}(u)\right\} \cap \underset{s_{1} s_{2} \ldots s_{n}=u^{\prime}, v^{\prime}}{\cup}\left\{\widehat{\mathscr{F}}^{\mathscr{H}}\left(u^{\prime}\right) \cap \widehat{\mathscr{F}}^{n-1}\left(v^{\prime}\right)\right\}\right\} \\
& \supseteq\left\{\left\{\widehat{\mathscr{F}}^{m}\left(r_{1} r_{2} \ldots r_{m}\right) \cap{\widehat{\mathscr{F}_{S}^{\mathscr{H}}}}_{(z)}\right\} \cap\left\{\widehat{\mathscr{F}}^{\mathscr{H}}\left(s_{1}\right) \cap \widehat{\mathscr{F}}^{n-1}\left(s_{2} \ldots y_{n-1} s_{n}\right)\right\}\right\} \\
& \supseteq\left\{\widehat{\mathscr{F}}^{m}\left(r_{1} r_{2} \ldots r_{m}\right) \cap \widehat{\mathscr{F}}^{n-1}\left(s_{1} s_{2} \ldots y_{n-1}\right) \cap \widehat{\mathscr{F}}^{\mathscr{H}}\left(s_{n}\right)\right\} \\
& \supseteq \widehat{\mathscr{F}^{\mathscr{H}}}\left(r_{1}\right) \cap \widehat{\mathscr{F}^{\mathscr{H}}}\left(r_{2}\right) \cap \cdots \cap \widehat{\mathscr{F}^{\mathscr{H}}}\left(r_{m}\right) \cap \widehat{\mathscr{F}^{\mathscr{H}}}\left(s_{1}\right) \cap \widehat{\mathscr{F}^{\mathscr{H}}}\left(s_{2}\right) \cap \cdots \cap \widehat{\mathscr{F}^{\mathscr{H}}}\left(s_{n}\right) .
\end{aligned}
$$

Hence, $\widehat{\mathscr{F}} \in \widehat{\mathscr{H}} \widehat{\mathscr{H}(m, n)}$.

Lemma 3. Let $\widehat{\mathscr{F}^{\mathscr{H}}}$ be the HFS of $S$. Then, $\widehat{\mathscr{F}^{\mathscr{H}}} \in \widehat{\wp_{\mathscr{H}_{B}}} \Rightarrow \widehat{\mathscr{F}^{\mathscr{H}}} \in \widehat{\wp_{\mathscr{H}(m, n)}} \forall m, n \in \mathbb{N}$. 


\begin{tabular}{lllll}
\hline & $\vartheta$ & $\iota$ & $\kappa$ & \\
\hline$\vartheta$ & $\vartheta$ & $\vartheta$ & $\vartheta$ & $\ddots$ \\
$\iota$ & $\iota$ & $\iota$ & $\iota$ & \\
$\kappa$ & $\kappa$ & $\kappa$ & $\kappa$ & $\kappa$ \\
$\hbar$ & $\vartheta$ & $\vartheta$ & $\iota$ & $\vartheta$ \\
\hline
\end{tabular}

Proof. Straightforward.

Remark 2. In general, $\widehat{\mathscr{F}^{\mathscr{H}}} \in \wp_{\mathscr{H}(m, n)} \nRightarrow \widehat{\mathscr{F}^{\mathscr{H}}} \in \widehat{\wp_{\mathscr{H}_{B}}}$.

Example 2. Let $S=\{\vartheta, \iota, \kappa, \hbar\}$ be a semigroup with the following multiplication table:

Define the HFS $\widehat{\mathscr{F}^{\mathscr{H}}}$ of $S$ as follows: $\widehat{\mathscr{F}^{\mathscr{H}}}(\vartheta)=\widehat{\mathscr{F}}^{\mathscr{H}}(\hbar)=$ $[0,1]$ and $\widehat{\mathscr{F}^{\mathscr{H}}}(\iota)=\widehat{\mathscr{F}^{\mathscr{H}}}(\hbar)=0$. Then, $\widehat{\mathscr{F}^{\mathscr{H}}} \in \wp_{\mathscr{H}(m, n)}$, but $\widehat{\mathscr{F}^{\mathscr{H}}} \notin \widehat{\wp_{\mathscr{H}_{B}}}$.

Definition 3. A semigroup $S$ is called $(m, n)$-regular if $\forall \hbar \in S \exists \ell \in S$ such that $\hbar=\hbar^{m} \ell \hbar^{n}$.

Lemma 4. Let $\widehat{\mathscr{F}^{\mathscr{H}}}$ be the HFS of $(m, n)$-regular semigroup $S$. Then, $\widehat{\mathscr{F}^{\mathscr{H}}} \in \widehat{\wp_{\mathscr{H}(m, n)}} \Rightarrow \widehat{\mathscr{F}^{\mathscr{H}}} \in \widehat{\wp_{\mathscr{H}_{B}}}$.
Proof. Suppose that $\widehat{\mathscr{F}^{\mathscr{H}}} \in \wp_{\mathscr{H}(m, n)}$ and $r, \hbar, s \in S$. Since $S$ is $(m, n)$-regular, $r \hbar s=r^{m} p r^{n} \hbar s^{m} q s^{n}$ for some $p, q \in S$. We have

$$
\begin{aligned}
& \widehat{\mathscr{F}^{\mathscr{H}}}(r \hbar s)=\widehat{\mathscr{F}^{\mathscr{H}}}\left(r^{m} p r^{n} \hbar s^{m} q s^{n}\right)=\widehat{\mathscr{F}^{\mathscr{H}}}\left(r^{m}\left(p r^{n} \hbar s^{m} q\right) s^{n}\right) \\
& \supseteq \widehat{\mathscr{F}}^{\mathscr{H}}(r) \cap \widehat{\mathscr{F}}^{\mathscr{H}}(s) \text {, }
\end{aligned}
$$

as required.

Lemma 5. Let $\widehat{\mathscr{F}^{\mathscr{H}}}$ be the HFS. Then, $\widehat{\mathscr{F}}^{\mathscr{H}}(\hbar) \subseteq \widehat{\mathscr{F}}^{k}\left(\hbar^{k}\right)$ for any $k \in \mathbb{N}$ and $\hbar \in S$.

Proof. Let $\hbar \in S$. As $\hbar^{k}=\hbar \hbar^{k-1}$, we have

$$
\begin{aligned}
\widehat{\mathscr{F}}^{\mathscr{H}}\left(\hbar^{k}\right) & =\hbar_{\hbar^{k}=u v}\left\{f(u) \cap \widehat{\mathscr{F}}^{k-1}(v)\right\} \supseteq \widehat{\mathscr{F}}^{\mathscr{H}}(\hbar) \cap \widehat{\mathscr{F}}^{k-1}\left(\hbar^{k-1}\right) \\
& =\widehat{\mathscr{F}}^{\mathscr{H}}(\hbar) \cap \underset{\hbar^{k-1}=u^{\prime} v^{\prime}}{\cup}\left\{\widehat{\mathscr{F}}^{\mathscr{H}}\left(u^{\prime}\right) t \cap \widehat{\mathscr{F}}^{k-2} q\left(v^{\prime}\right)\right\} \supseteq \widehat{\mathscr{F}}^{\mathscr{H}}(\hbar) \cap \widehat{\mathscr{F}}^{\mathscr{H}}(\hbar) \cap \widehat{\mathscr{F}}^{k-2}\left(\hbar^{k-2}\right) \\
& \vdots \\
& \supseteq \widehat{\mathscr{F}}^{\mathscr{H}}(\hbar) \cap \cdots \cap \widehat{\mathscr{F}}^{\mathscr{H}}(\hbar) \cap \widehat{\mathscr{F}}^{\mathscr{H}}(\hbar)=\widehat{\mathscr{F}}^{\mathscr{H}}(\hbar) .
\end{aligned}
$$

Theorem 3. S is $(m, n)$-regular $\Leftrightarrow \widehat{\mathscr{F}}^{\mathscr{H}} \subseteq \widehat{\mathscr{F}}^{m} \widehat{\mathscr{I}}_{S}^{\mathscr{H}} \circ \widehat{\mathscr{F}}^{n}$ for $\quad$ Proof. $\quad(\Rightarrow)$ Take any $\hbar \in S$. Then, $\hbar=\hbar^{m} \ell \hbar^{n}$ for some $\ell \in S$. each HFS $\mathscr{F}^{\mathscr{H}}$ of $S$. We have

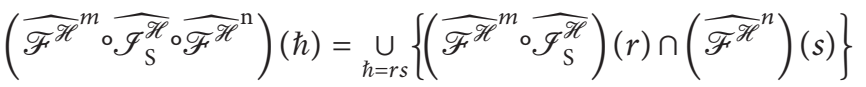

$$
\begin{aligned}
& \left.\supseteq\left(\widehat{\mathscr{F}}^{m} \widehat{\mathscr{I}}_{\mathrm{S}}^{\mathscr{H}}\right)\left(\hbar^{m} x\right) \cap\left(\widehat{\mathscr{F}}^{n}\right)\left(\hbar^{n}\right)={ }_{\hbar^{m} x=p q}^{\cup}\left\{\left(\widehat{\mathscr{F}}^{m}\right)(p) \cap{\widehat{\mathscr{J}_{S}^{\mathscr{H}}}}^{m} q\right)\right\} \cap\left(\widehat{\mathscr{F}}^{n}\right)\left(\hbar^{n}\right)
\end{aligned}
$$

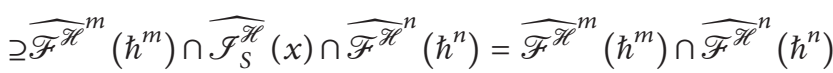

$$
\begin{aligned}
& \supseteq \widehat{\mathscr{F}}^{\mathscr{H}}(\hbar) \cap \widehat{\mathscr{F}^{\mathscr{H}}}(\hbar) \text { by Lemma } 2.14=\widehat{\mathscr{F}^{\mathscr{H}}}(\hbar) \text {. }
\end{aligned}
$$


Therefore, $\widehat{\mathscr{F}}^{\mathscr{H}} \subseteq \widehat{\mathscr{F}}^{m} \circ \widehat{\mathscr{J}}_{\mathrm{S}} \circ \widehat{\mathscr{F}}^{\mathrm{H}}$.

$(\Leftarrow)$ Suppose that $\hbar \in S$. Since $\widehat{\chi_{\hbar}^{\mathscr{H}}}$ is the HFS of $S$, by

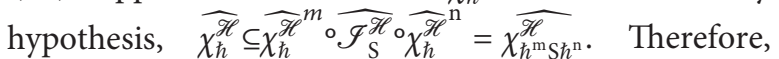
$\hbar \in \hbar^{m} S \hbar^{n}$. Hence, $S$ is $(m, n)$-regular. $\square$

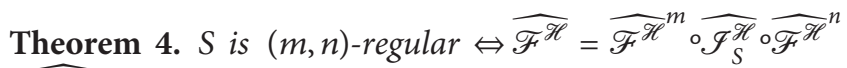
$\forall \widehat{\mathscr{F}^{\mathscr{H}}} \in \widehat{\wp} \widehat{\mathscr{H}(m, n)}$

Proof. $(\Rightarrow)$ Let $\widehat{\mathscr{F}^{\mathscr{H}}} \in \wp_{\mathscr{\mathscr { H }}(m, n)}$. Then, by hypothesis and Theorems 2 and 4, $\widehat{\mathscr{F}}^{m} \circ{\widehat{\mathscr{F}_{\mathrm{S}}}}^{\circ} \widehat{\mathscr{F}}^{\mathscr{H}} \mathrm{\textrm {n }} \subseteq \widehat{\mathscr{F}}^{\mathscr{H}}$ and $\widehat{\mathscr{F}}^{\mathscr{H}} \subseteq \widehat{\mathscr{F}}^{m} \circ \widehat{\mathscr{F}}_{\mathrm{S}}^{\mathscr{H}} \circ \widehat{\mathscr{F}}^{\mathrm{H}}$. $\widehat{\mathscr{F}^{\mathscr{H}}}=\widehat{\mathscr{F}}^{m} \circ \widehat{\mathscr{S}}_{\mathrm{S}}^{\mathscr{H}} \circ \widehat{\mathscr{F}}^{\mathrm{H}} \mathrm{n}$.

Hence,

$(\Leftarrow)$ Let $B \in \mathscr{I}_{(m, n)}$ and $b \in B$, so by hypothesis, we have $\quad\left(\widehat{\chi}_{B}^{m} \circ \mathscr{I}_{\mathrm{S}}^{\mathscr{H}_{\mathrm{H}}} \circ{\widehat{\chi_{\mathrm{B}}}}^{\mathrm{n}}\right)(\mathrm{b})=\chi_{\mathrm{A}}(\mathrm{b})=1 \quad$ implies $\left({\widehat{\chi_{B}}}^{m} \circ{\widehat{\mathscr{F}_{\mathrm{S}}}}^{\mathscr{H}} \circ \widehat{\chi}_{\mathrm{B}}^{\mathscr{F}^{\mathrm{n}}}\right)(\mathrm{b})=1$. This implies that there exist elements $\hbar, \kappa$ in $S$ with $b=\hbar \kappa$ such that

$$
\left({\widehat{\chi_{B}^{\mathscr{H}}}}^{m} \circ{\widehat{\mathscr{Y}_{\mathrm{S}}^{\mathscr{H}}}}^{\prime}(\hbar)=[0,1] \text { and }{\widehat{\chi_{B}}}^{n}(\kappa)=[0,1]\right.
$$

This implies that there exist elements $u, v$ in $S$ with $\hbar=$ $u v$ such that

$$
{\widehat{\chi_{B}^{\mathscr{H}}}}^{m}(u)=[0,1] \text { and } \widehat{\mathscr{F}_{S}^{\mathscr{H}}}(v)=[0,1]
$$

So, $\quad[0,1]={\widehat{\chi_{B}^{\mathscr{H}}}}^{n}(\kappa)=\widehat{\chi_{B^{n}}}(\kappa) \quad$ and $[0,1]={\widehat{\chi_{B}^{\mathscr{H}}}}^{m}(u)=\widehat{\chi_{B^{m}}}(u)$, and it follows that $\kappa \in B^{n}$ and $u \in B^{n}$. Since $a=\hbar \kappa$ and $\hbar=u v$, therefore, $a=\hbar \kappa=u v \kappa \in B^{m} S B^{n}$. Thus, $B \subseteq B^{m} S B^{n}$. Therefore, $B=B^{m} S B^{n}$. Hence, by Theorem 2of [44], $S$ is $(m, n)$-regular.

Lemma 6. If $\widehat{\mathscr{F}^{\mathscr{H}}} \in \widehat{\wp_{\mathscr{H}(m, n)}}$ and $\widehat{\mathscr{G}^{\mathscr{H}}}$ is a HFSS of S such that

$$
{\widehat{\mathscr{F}^{\mathscr{H}}}}^{m} \circ{\widehat{\mathscr{F}_{\mathrm{S}}^{\mathscr{H}}}}_{\widehat{\mathscr{F}}^{\mathscr{H}}}^{\mathrm{n}} \subseteq \widehat{\mathscr{G}}^{\mathscr{H}} \subseteq \widehat{\mathscr{F}^{\mathscr{H}}} \text {, }
$$

then $\widehat{\mathscr{G}^{\mathscr{H}}} \in \widehat{\wp} \widehat{\mathscr{H}(m, n)}$.

Proof. Since $\widehat{\mathscr{G}^{\mathscr{H}}}$ is a HFSS of $S$, by Theorem 2, it is sufficient to show that $\widehat{\mathscr{G}^{\mathscr{H}}} \widehat{\circ}_{\mathscr{F}_{\mathrm{S}}^{\mathscr{H}}}^{\circ \mathscr{G}^{\mathscr{H}}} \subseteq \widehat{\mathscr{G}^{\mathscr{H}}}$. Now,

$$
\left(\widehat{\mathscr{G}}^{\mathscr{H}^{m}} \circ \widehat{\mathscr{F}}_{\mathrm{S}}^{\mathscr{H}} \circ \widehat{\mathscr{G}}^{\mathscr{H}} \mathrm{n}\right)(a) \subseteq\left(\widehat{\mathscr{F}}^{m} \circ{\widehat{\mathscr{F}_{\mathrm{S}}^{\mathscr{H}}}}_{\mathscr{F}^{\mathscr{H}}}^{\mathrm{n}}\right)(a) \subseteq \widehat{\mathscr{G}}^{\mathscr{H}}(a) .
$$

Hence, $\widehat{\mathscr{G}^{\mathscr{H}}} \in \widehat{\wp} \widehat{\mathscr{H}(m, n)}$.

Lemma 7. Let $\widehat{\mathscr{F}^{\mathscr{H}}} \in \widehat{\wp_{\mathscr{H}(m, n)}}$ and $\widehat{\mathscr{G}^{\mathscr{H}}}$ be a HFS of S. If $\widehat{\mathscr{F}^{\mathscr{H}}} \circ \widehat{\mathscr{G}^{\mathscr{H}}} \widehat{\widehat{F}^{\mathscr{H}}}$ or $\widehat{\mathscr{G}^{\mathscr{H}}} \circ \widehat{\mathscr{F}}^{\mathscr{H}} \subseteq \widehat{\mathscr{F}^{\mathscr{H}}}$, then
(1) $\widehat{\mathscr{F}^{\mathscr{H}}} \circ \widehat{\mathscr{G}^{\mathscr{H}}} \in \wp \widehat{\mathscr{H}(m, n)}$.
(2) $\widehat{\mathscr{G}^{\mathscr{H}}} \widehat{\mathscr{F}^{\mathscr{H}}} \in \wp \widehat{\mathscr{H}(m, n)}$.

Proof. When $\widehat{\mathscr{F}^{\mathscr{H}}} \circ \widehat{\mathscr{G}^{\mathscr{H}}} \subseteq \widehat{\mathscr{F}}$, we have

$$
\left(\widehat{\mathscr{F}^{\circ}} \cdot \widehat{\mathscr{G}^{\mathscr{H}}}\right) \circ\left(\widehat{\mathscr{F}^{\mathscr{H}}} \widehat{\mathscr{G}^{\mathscr{H}}}\right)=\widehat{\mathscr{F}^{\mathscr{H}}} \mathrm{\circ}\left(\widehat{\mathscr{F}^{\mathscr{H}}} \mathrm{\circ}, \widehat{\mathscr{G}^{\mathscr{H}}}\right)=\widehat{\mathscr{F}^{\mathscr{H}}} \cdot \widehat{\mathscr{F}^{\mathscr{H}}} \circ \widehat{\mathscr{G}^{\mathscr{H}}} \subseteq \widehat{\mathscr{F}^{\mathscr{H}}} \circ \widehat{\mathscr{G}^{\mathscr{H}}} .
$$

Therefore, $\widehat{\mathscr{F}^{\mathscr{H}}} \circ \widehat{\mathscr{G}^{\mathscr{H}}}$ is a HFSS of $S$. Also, we have

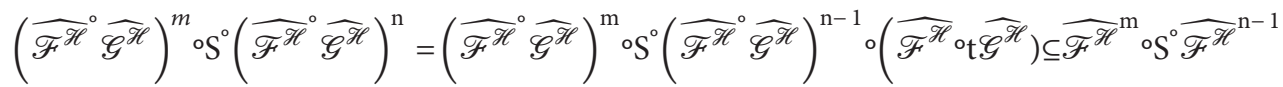

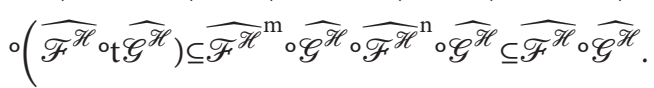

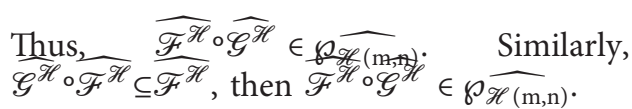

(2)Similar to (1).

\section{Hesitant Fuzzy $(m, 0)$-Ideals and Hesitant Fuzzy $(0, n)$-Ideals}

Definition 4. A HFSS $\widehat{\mathscr{F}^{\mathscr{H}}}$ of $S$ is called a hesitant fuzzy $(m, 0)$-ideal of $S$ if when

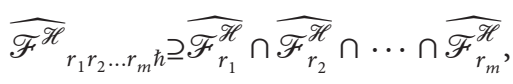

for all $r_{1}, r_{2}, \ldots, r_{m}, \hbar \in S$.

Dually, a hesitant fuzzy $(0, n)$-ideal of $S$ can be defined.
Lemma 8. Let $\widehat{\mathscr{F}^{\mathscr{H}}}$ be the HFS of S. Then, $\widehat{\mathscr{F}^{\mathscr{H}}} \in \widehat{\wp_{\mathscr{H}}}$ (resp. $\left.\widehat{\mathscr{F}^{\mathscr{H}}} \in \widehat{\wp_{\mathscr{H}}}\right) \Rightarrow \widehat{\mathscr{F}^{\mathscr{H}}} \in \widehat{\wp_{\mathscr{H}(m, 0)}} \quad\left(\right.$ resp. $\left.\quad \widehat{\mathscr{F}^{\mathscr{H}}} \in \widehat{\wp_{\mathscr{H}(0, n)}}\right)$ $\forall m, n \in \mathbb{N}$.

Proof. Straightforward. 
Remark 3. In general, converse of Lemma 8 does not hold.

Example 3. In Example 2, the HFS $\widehat{\mathscr{F}^{\mathscr{H}}} \in \widehat{\wp_{\mathscr{H}(m, 0)}}, \widehat{\wp_{\mathscr{H}(0, n)}} \forall m, n \in \mathbb{N}, m, n \geq 2, \quad$ but $\widehat{\mathscr{F}^{\mathscr{H}}} \notin \widehat{\wp_{\mathscr{H}_{R}}}, \widehat{\wp_{\mathscr{H}_{L}}}$

Definition 5. A semigroup $S$ is called $(m, 0)$-regular (resp. $(0, n)$-regular) if $\forall \hbar \in S \exists \kappa \in S$ such that $\hbar=\hbar^{m} \kappa\left(\right.$ resp. $\left.\hbar=\kappa \hbar^{n}\right)$.

Lemma 9. In $S$, the following assertions hold:

$$
\begin{aligned}
& \text { (1) In }(m, 0) \text {-regular semigroup } \\
& \widehat{\mathscr{F}^{\mathscr{H}}} \in \widehat{\wp \mathscr{H}(m, 0)} \Rightarrow \widehat{\mathscr{F}^{\mathscr{H}}} \in \widehat{\wp_{\mathscr{H}_{R}}} \\
& \text { (2) } \frac{\text { In }}{\widehat{\mathscr{F}}^{\mathscr{H}}} \in \widehat{(0, n) \text {-regular }} \widehat{\widehat{\wp_{\mathscr{H}}(0, n)}} \Rightarrow \widehat{\mathscr{F}^{\mathscr{H}}} \in \widehat{\wp_{\mathscr{H}}} \\
& \text { semigroup }
\end{aligned}
$$

Proof. Let $\widehat{\mathscr{F}^{\mathscr{H}}} \in \widehat{\wp \mathscr{H}(m, 0)}$ and $\hbar, \kappa \in S$. Since $S$ is $(m, 0)$-regular, $\exists \ell \in S$ such that $\hbar \kappa=\hbar^{m} \ell \kappa$. Therefore, we have

$$
\widehat{\mathscr{F}^{\mathscr{H}}}(\hbar \kappa)=\widehat{\mathscr{F}^{\mathscr{H}}}\left(\hbar^{m} \ell \kappa\right)=\widehat{\mathscr{F}^{\mathscr{H}}}\left(\hbar^{m}(\ell \kappa)\right) \supseteq \widehat{\mathscr{F}}^{\mathscr{H}}(\hbar) .
$$

Hence, $\widehat{\mathscr{F}^{\mathscr{H}}} \in \widehat{\wp_{\mathscr{H}_{R}}}$.

(2) Similar to the proof of (1).

Lemma 10. Let $\varnothing \neq \Omega \subseteq S$. Then, $\quad \Omega \in \mathscr{I}_{(m, 0)}$ (resp. $\left.\Omega \in \mathscr{I}_{(0, n)}\right) \Leftrightarrow H F S \widehat{\chi_{\Omega}^{\mathscr{H}}} \in \wp_{\mathscr{H}(m, 0)}\left(\operatorname{resp} \cdot \widehat{\chi_{\Omega}^{\mathscr{H}}} \in \wp_{\mathscr{H}(0, n)}\right)$.

Proof. $\quad(\Rightarrow)$ Let $r_{1}, r_{2}, \ldots, r_{m}, z \in S$. If $x_{i} \notin \Omega$ for any $i \in\{1,2, \ldots, m\}$, then

$\widehat{\chi_{\Omega}}\left(r_{1} r_{2} \ldots r_{m} z\right) \supseteq \widehat{\chi_{\Omega}}\left(r_{1}\right) \cap \widehat{\chi_{\Omega}}\left(r_{2}\right) \cap \cdots \cap \widehat{\chi_{\Omega}}\left(r_{m}\right)$.

If $r_{i} \in \Omega$ for each $i \in\{1,2, \ldots, m\}$, then $r_{1} r_{2} \cdots r_{m} z \in \Omega^{m} S \subseteq \Omega$. Therefore,

$$
\widehat{\chi_{\Omega}^{\mathscr{H}}}\left(r_{1} r_{2} \ldots r_{m} z\right)=1 \supseteq \widehat{\chi_{\Omega}}\left(r_{1}\right) \cap \widehat{\chi_{\Omega}^{\mathscr{H}}}\left(r_{2}\right) \cap \cdots \cap \widehat{\chi_{\Omega}}\left(r_{m}\right) .
$$

Hence, $\Omega \in \mathscr{I}_{(m, 0)}$.

$(\Leftarrow)$ Suppose that $x, z \in S$. If $x \in \Omega$, then $\widehat{\chi_{\Omega}^{\mathscr{H}}}\left(x^{m} z\right) \supseteq \widehat{\chi_{\Omega}}(x)=1$ implies $\widehat{\chi_{\Omega}^{\mathscr{H}}}\left(x^{m} z\right)=1$. Therefore, $x^{m} z \in \Omega$. Thus, $\Omega^{m} S \subseteq \Omega$.

Theorem 5. Let $\widehat{\mathscr{G}^{\mathscr{H}}}$ be the HFS of S. Then, $\left[\widehat{\mathscr{G}}^{\mathscr{H}}\right]_{T} \in \mathscr{I}_{(m, 0)}$ (resp. $\left.\left[\widehat{\mathscr{G}^{\mathscr{H}}}\right]_{T} \in \mathscr{I}_{(m, 0)}\right) \quad \forall T \in \mathscr{P}([0,1]), \quad$ provided $\left[\widehat{\mathscr{G}^{\mathscr{H}}}\right]_{T} \neq \varnothing \Leftrightarrow \widehat{\mathscr{G}^{\mathscr{H}}} \in \widehat{\wp_{\mathscr{H}(m, 0)}}$ (resp. $\widehat{\mathscr{G}^{\mathscr{H}}} \in \widehat{\wp_{\mathscr{H}(0, n)}}$.

Proof. $\quad(\Rightarrow)$ Suppose that $z \in S$ and $r_{1}, r_{2}, \ldots, r_{m} \in \widehat{\mathscr{G}_{T}^{\mathscr{H}}}$, where $\quad T \in \mathscr{P}([0,1]) . \quad$ Then, $\widehat{\mathscr{G}_{r_{1}}^{\mathscr{H}}} \supseteq T, \widehat{\mathscr{G}_{r_{2}}^{\mathscr{H}}} \supseteq T, \ldots, \widehat{\mathscr{G}_{r_{m}}^{\mathscr{H}}} \supseteq T$. By Definition 4, $\mathscr{G}_{r_{1} r_{2} \cdots r_{m} z}^{\widehat{\mathscr{H}}} \supseteq \widehat{\mathscr{G}_{r_{1}}^{\mathscr{H}}} \cap \widehat{\mathscr{G}_{r_{2}}^{\mathscr{H}}} \cap \cdots \cap \widehat{\mathscr{G}_{r_{m}}^{\mathscr{H}}}=T . \quad$ Therefore, $r_{1} r_{2} \cdots r_{m} z \in\left[\widehat{\mathscr{G}^{\mathscr{H}}}\right]_{T}$. Hence, $\left[\widehat{\mathscr{G}^{\mathscr{H}}}\right]_{T} \in \mathscr{I}_{(m, n)}$. $(\Leftarrow)$ Let $\left[\widehat{\mathscr{G}}^{\mathscr{H}}\right]_{T} \in \mathscr{I}_{(m, 0)} \forall T \in \mathscr{P}([0,1])$ and $x, y \in S$. Suppose, to the contrary, that $\widehat{\mathscr{G}_{r_{1}}^{\mathscr{H}}} \cap \widehat{\mathscr{G}_{r_{2}}^{\mathscr{H}}} \cap \cdots \cap{\widehat{\mathscr{G}_{r_{m}}^{\mathscr{H}}}}_{\mathscr{G}_{r_{1} r_{2} \cdots r_{m}}}^{\widehat{S}}$. Then, there exists $C \in \mathscr{P}([0,1]) \quad$ such that $\widehat{\mathscr{G}_{r_{1}}^{\mathscr{H}}} \cap \widehat{\mathscr{G}_{r_{2}}^{\mathscr{H}}} \cap \cdots \cap \widehat{\mathscr{G}_{r_{m}}^{\mathscr{H}}} \supseteq C^{\widehat{\mathscr{G}_{r_{1} r_{2} \cdots r_{m} z}}}$. This implies that $r_{1}, r_{2}, \ldots, r_{m}, z, \in\left[\widehat{\mathscr{G}^{\mathscr{H}}}\right]_{C}$, but $r_{1} r_{2} \cdots r_{m} z \notin\left[\widehat{\mathscr{G}^{\mathscr{H}}}\right]_{C}$, a contradiction. Thus, $\mathscr{G}_{r_{1} r_{2} \cdots r_{m} z}^{\mathscr{\mathscr { H }}} \supseteq \widehat{\mathscr{G}_{r_{1}}^{\mathscr{H}}} \cap \widehat{\mathscr{G}_{r_{2}}^{\mathscr{H}}} \cap \cdots \cap \widehat{\mathscr{G}_{r_{m}}^{\mathscr{H}}}$ for all $r_{1}, r_{2}, \ldots, r_{m}, z \in S$. Hence, by Definition $4, \widehat{\mathscr{G}^{\mathscr{H}}}$ is a hesitant fuzzy $(m, 0)$-ideal of $S$.

Theorem 6. Let $\widehat{\mathscr{F}^{\mathscr{H}}}$ be any HFSS of S. Then, $\widehat{\mathscr{F}^{\mathscr{H}}} \in \wp_{\mathscr{H}(m, 0)}$ (resp. $\left.\quad \widehat{\mathscr{F}}^{\mathscr{H}} \in \widehat{\wp_{\mathscr{H}(0, n)}}\right) \quad \Leftrightarrow \widehat{\mathscr{F}}^{m}{\widehat{\mathscr{F}_{S}^{\mathscr{H}}}} \subseteq \widehat{\mathscr{F}}^{\mathscr{H}} \quad$ (resp. \left.${\widehat{\mathscr{I}_{S}^{\mathscr{H}}}}_{\mathscr{\mathscr { F }}^{\mathscr{H}}}^{n} \subseteq \widehat{\mathscr{F}}^{\mathscr{H}}\right)$.

Proof. On the similar lines to the proof of Theorem 2.

Lemma 11. If $S$ is an $(m, n)$-regular semigroup, then

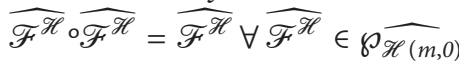
and $\widehat{\mathscr{G}^{\mathscr{H}}} \circ \widehat{\mathscr{G}^{\mathscr{H}}}=\widehat{\mathscr{G}^{\mathscr{H}}} \forall \widehat{\mathscr{G}^{\mathscr{H}}} \in \widehat{\wp_{\mathscr{H}(0, n)}}$.

Proof. Let $S$ be an $(m, n)$-regular semigroup and $\widehat{\mathscr{F}^{\mathscr{H}}} \in \widehat{\wp_{\mathscr{H}(m, 0)}}$. Then, $\widehat{\mathscr{F}^{\mathscr{H}}} \circ \widehat{\mathscr{F}^{\mathscr{H}}} \subseteq \widehat{\mathscr{F}^{\mathscr{H}}}$. As $S$ is $(m, n)$-regular, we have

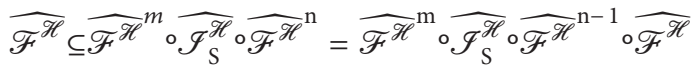

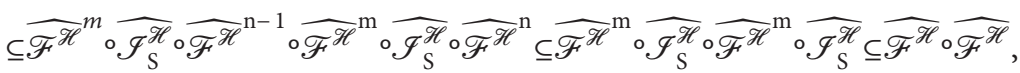


and so, we obtain $\widehat{\mathscr{F}^{\mathscr{H}}} \subseteq \widehat{\mathscr{F}^{\mathscr{H}}} \circ \widehat{\mathscr{F}}^{\mathscr{H}}$. Hence, $\widehat{\mathscr{F}^{\mathscr{H}}}=\widehat{\mathscr{F}^{\mathscr{H}}} \circ \widehat{\mathscr{F}}^{\mathscr{H}}$. Similarly, we may prove that $\widehat{\mathscr{G}^{\mathscr{H}}} \circ \widehat{\mathscr{G}^{\mathscr{H}}}=\widehat{\mathscr{G}^{\mathscr{H}}} \forall \widehat{\mathscr{G}^{\mathscr{H}}} \in \widehat{\wp_{\mathscr{H}(0, \mathrm{n})}}$.

Theorem 7. The following statements hold in S:

(1) $\underline{S \text { is }}(m, 0)$-regular $\Leftrightarrow \widehat{\mathscr{F}}^{\mathscr{H}} \subseteq \widehat{\mathscr{F}}^{m} \widehat{\circ}^{\mathscr{H}}$ for each HFS $\mathscr{F}^{\mathscr{H}}$ of $S$ (2) $\frac{S \text { is }}{\mathscr{F}_{\text {H }} S}(0, n)$-regular $\Leftrightarrow \widehat{\mathscr{F}}^{\mathscr{H}} \subseteq{\widehat{\mathscr{F}_{S}^{\mathscr{H}}}}_{\circ \mathscr{F}^{\mathscr{H}}}{ }^{n}$ for each HFS

Proof

(1) $(\Rightarrow)$ Take any $\hbar \in S$. Then, $\exists \kappa \in S$ such that $\hbar=\hbar^{m} \kappa$. Now, we have

$$
\begin{aligned}
\left(\widehat{\mathscr{F}}^{m} \circ \widehat{\mathscr{I}}_{\mathrm{S}}^{\mathscr{H}}\right)(\hbar) & =\bigcup_{\hbar=r s}\left\{\left(\widehat{\mathscr{F}}^{m}\right)(r) \cap{\widehat{\mathscr{I}_{S}^{\mathscr{H}}}}^{m}(s)\right\} \supseteq\left(\widehat{\mathscr{F}}^{m}\right)\left(\hbar^{m}\right) \cap{\widehat{\mathscr{I}_{S}^{\mathscr{H}}}}^{m}(\kappa) \\
& =\left(\widehat{\mathscr{F}}^{m}\right)\left(\hbar^{m}\right)=\widehat{\mathscr{F}}^{\mathscr{H}}(\hbar) .
\end{aligned}
$$

Therefore, $\widehat{\mathscr{F}}^{\mathscr{H}} \subseteq \widehat{\mathscr{F}}^{m} \circ \widehat{\mathscr{I}}_{\mathrm{S}}^{\mathscr{H}}$.

$(\Leftarrow)$ Let $\hbar \in S$. Since $\widehat{\chi_{\hbar}^{\mathscr{H}}}$ is the HFS of $S$, by hypothesis, $\quad \widehat{\chi_{\hbar}^{\mathscr{H}}} \subseteq{\widehat{\chi_{\hbar}}}^{m} \circ{\widehat{\mathscr{F}_{\mathrm{S}}^{\mathscr{H}}}}^{\circ}{\widehat{\chi_{\hbar}^{\mathscr{H}}}}^{\mathrm{n}}=\mathscr{H}_{\hbar^{\mathrm{m}} \mathrm{S} \hbar^{\mathrm{n}}}$. So, $\hbar \in \hbar^{m} S \hbar^{n}$, and hence, $S$ is $(m, n)$-regular.

(2) Similar to the proof of (1).

Theorem 8. The following assertions are true in $S$ :

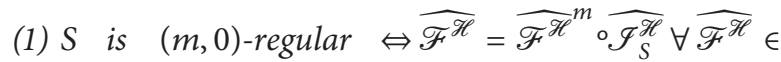
$\widehat{\wp \mathscr{H}(m, 0)}$

(2) S is $(0, n)$-regular $\Leftrightarrow \widehat{\mathscr{G}^{\mathscr{H}}}=\widehat{\mathscr{G}_{S}^{\mathscr{H}}} \circ \widehat{\mathscr{G}}^{n} \forall \widehat{\mathscr{G}^{\mathscr{H}}} \in \widehat{\wp_{\mathscr{H}(0, n)}}$

\section{Proof}

(1) $(\Rightarrow)$ Let $\widehat{\mathscr{F}^{\mathscr{H}}} \in \wp_{\mathscr{\mathscr { H }}(m, 0)}$. Then, by hypothesis and Theorems 7 and 6, we have $\widehat{\mathscr{F}}^{\mathscr{H}} \subseteq \widehat{\mathscr{F}}^{m}{\widehat{\mathscr{F}_{\mathrm{S}}^{\mathscr{H}}}}$ and $\widehat{\mathscr{F}}^{m} \circ{\widehat{\mathscr{I}_{\mathrm{S}}^{\mathscr{H}}}}_{\widehat{\mathscr{F}^{\mathscr{H}}}}$. Hence, $\widehat{\mathscr{F}}^{\mathscr{H}}=\widehat{\mathscr{F}}^{m} \circ{\widehat{\mathscr{I}_{\mathrm{S}}^{\mathscr{H}}}}$.

$(\Leftarrow)$ Let $R$ be any $(m, 0)$-ideal of $S$, and take $a \in R$. Then, by hypothesis, we have $\left(\mathscr{H}_{R}^{m_{0}}\right.$ $\left.\mathscr{I}_{S}^{\mathscr{H}}\right)(a)=\mathscr{H}_{R}(a)=[0,1]$ implies $\left(\mathscr{H}_{R}^{m_{\circ}} \widehat{\mathscr{I}}_{\mathrm{S}}^{\mathscr{H}}\right)(\mathrm{a})^{R}=$ $[0,1]$. Therefore, there exist elements $x, y$ in $S$ with $\underline{a=x y}$ such that $\mathscr{H}_{R}^{m}(x)=[0,1]$ and $\mathscr{I}_{S}^{\mathscr{H}}(y)=[0,1]$. As we have $\mathscr{H}_{R}^{m}(x)=[0,1]$, $\mathscr{H}_{R^{m}}(x)=[0,1]$, and it follows that $x \in R^{m}$. Since $a=x y$, therefore, $a=x y \in R^{m} S$. Thus, $R \subseteq R^{m} S$.
Since $R$ is $(m, 0)$-ideal of $S, R^{m} S \subseteq R$. Therefore, $R=R^{m} S$. Hence, by Theorem 1 of [44], $S$ is $(m, 0)$-regular.

(2) Similar to the proof of (1).

Theorem 9. A semigroup $S$ is $(m, n)$-regular $\Leftrightarrow \widehat{\mathscr{F}^{\mathscr{H}}} \cap \widehat{\mathscr{G}^{\mathscr{H}}}=$ $\widehat{\mathscr{F}}^{m} \circ \widehat{\mathscr{G}}^{\mathscr{H}} \cap \widehat{\mathscr{F}^{\mathscr{H}}} \circ \widehat{\mathscr{G}}^{\mathscr{H}^{n}} \forall \widehat{\mathscr{F}^{\mathscr{H}}} \in \wp_{\mathscr{H}(m, 0)}$ and $\forall \widehat{\mathscr{G}^{\mathscr{H}}} \in \widehat{\wp_{\mathscr{H}(0, n)}}$.

Proof.

$(\Rightarrow)$ Let $\widehat{\mathscr{F}^{\mathscr{H}}} \in \widehat{\wp_{\mathscr{H}(m, 0)}}$ and $\widehat{\mathscr{G}^{\mathscr{H}}} \in \widehat{\wp_{\mathscr{H}(0, n)}}$. As $S$ is $(m, n)$-regular, we have

$\widehat{\mathscr{F}}^{\mathscr{H}} \cap \widehat{\mathscr{G}}^{\mathscr{H}} \subseteq\left(\widehat{\mathscr{F}}^{\mathscr{H}} \cap t \widehat{\mathscr{G}}^{\mathscr{H}}\right)^{m} \circ \widehat{\chi}^{\mathscr{H}} \circ\left(\widehat{\mathscr{F}}^{\mathscr{H}} \cap \mathrm{t} \widehat{\mathscr{G}}^{\mathscr{H}}\right)^{\mathrm{n}} \subseteq \widehat{\mathscr{F}}^{\mathrm{H}} \mathrm{m}$ $\widehat{\chi^{\mathscr{H}}} \circ \widehat{\mathscr{G}}^{\mathrm{H}} \subseteq \widehat{\mathscr{F}}^{\mathrm{H}} \circ \widehat{\mathscr{G}}^{\mathscr{H}}$, and so, $\widehat{\mathscr{F}}^{\mathscr{H}} \cap \widehat{\mathscr{G}}^{\mathscr{H}} \subseteq \widehat{\mathscr{F}}^{m} \circ \widehat{\mathscr{G}}^{\mathscr{H}}$. Similarly, $\quad \widehat{\mathscr{F}^{\mathscr{H}}} \cap \widehat{\mathscr{G}^{\mathscr{H}}} \subseteq \widehat{\mathscr{F}^{\mathscr{H}}} \circ \widehat{\mathscr{G}}^{\mathrm{H}} \mathrm{n}$. Thus, $\widehat{\mathscr{F}^{\mathscr{H}}} \cap$ $\widehat{\mathscr{G}^{\mathscr{H}}}{\widehat{\mathscr{F}^{\mathscr{H}}}}^{m} \circ \widehat{\mathscr{G}}^{\mathscr{H}} \cap \widehat{\mathscr{F}^{\mathscr{H}}} \circ \widehat{\mathscr{G}}^{\mathscr{H}^{\mathrm{n}}}$. As $\widehat{\mathscr{F}}_{\mathscr{H}} \in \widehat{\wp \mathscr{H}(m, 0)}$ and $\widehat{\mathscr{G}^{\mathscr{H}}} \in \widehat{\wp} \widehat{\mathscr{H}(0, n)}, \quad \widehat{\mathscr{F}}^{m} \circ \widehat{\mathscr{G}}^{\mathscr{H}} \cap \widehat{\mathscr{F}}^{\mathscr{H}} \circ \widehat{\mathscr{G}}^{\mathrm{H}} \subseteq \widehat{\mathscr{F}^{\mathscr{H}}} \cap \widehat{\mathscr{G}^{\mathscr{H}}}$. Therefore, $\widehat{\mathscr{F}^{\mathscr{H}}} \cap \widehat{\mathscr{G}}^{\mathscr{H}}=\widehat{\mathscr{F}}^{m} \circ \widehat{\mathscr{G}}^{\mathscr{H}} \cap \widehat{\mathscr{F}^{\mathscr{H}}} \circ \widehat{\mathscr{G}}^{\mathrm{H}} \mathrm{n}$.

$(\Leftarrow)$ Let $a \in R \cap L$ for $R \in \mathscr{I}_{(m, 0)}$ and $L \in \mathscr{I}_{(0, n)}$. By Lemma 10, $\widehat{\chi_{R}^{\mathscr{H}}} \in \widehat{\wp \mathscr{H}(m, 0)}$ and $\chi_{L}^{\mathscr{H}} \in \widehat{\wp_{\mathscr{H}(0, n)}}$. Therefore, by hypothesis,

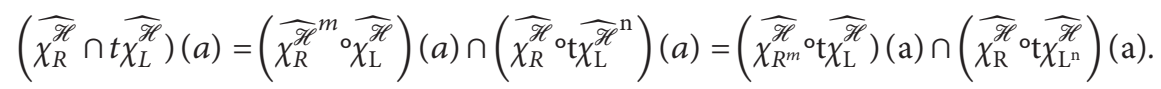

Since $\quad \mathscr{H}_{R}(a)=[0,1] \quad$ and $\mathscr{H}_{L}(a)$, $\left(\widehat{\chi_{R}^{\mathscr{H}}} \cap t \widehat{\chi_{L}^{\mathscr{H}}}\right)(a)=[0,1], \quad\left(\widehat{\chi}_{R^{m}}^{\mathscr{H}} \mathrm{ot} \widehat{\chi}_{\mathrm{L}}^{\widehat{\mathscr{H}}}\right)(\mathrm{a})=[0,1] \quad$ and $\left(\widehat{\chi_{R}^{\mathscr{H}}} \mathrm{o}^{\mathrm{t}}{\widehat{\mathrm{L}^{\mathrm{n}}}}^{\mathscr{\mathscr { L }}}\right)(\mathrm{a})=[0,1]$. This implies that there exist $x, y, u$, and $v$ in $S$ with $a=x y$ and $a=u v$ such that
$\widehat{\chi_{R^{m}}}(x)=[0,1], \widehat{\chi_{L^{n}}}(y)=[0,1]$, and $\widehat{\chi_{R}^{\mathscr{H}}}(u)=[0,1]$, $\widehat{\chi_{L^{n}}}(v)=[0,1]$, and it follows that $x \in R^{m}, y \in L$ and $u \in R, v \in L^{n}$. As $a=x y$ and $a=u v, a=x y \in R^{m} L$ and $a=u v \in R L^{n}$ imply $a \in R^{m} L \cap R L^{n}$. Thus, we obtain 
$R \cap L \subseteq R^{m} L \cap R L^{n}$. Also, $R^{m} L \cap R L^{n} \subseteq R \cap L$. Therefore, $R \cap L=R^{m} L \cap R L^{n}$. Hence, by Theorem 3 of [44], $S$ is $(m, n)$-regular.
Proposition 1. Let $\widehat{\mathscr{F}^{\mathscr{H}}} \in \widehat{\wp_{\mathscr{H}(m, 0)}}$ and $\widehat{\mathscr{G}^{\mathscr{H}}} \in \widehat{\wp_{\mathscr{H}(0, n)}}$. If $\widehat{\mathscr{F}^{\mathscr{H}}} \circ \widehat{\mathscr{G}^{\mathscr{H}}}=\widehat{\mathscr{G}}^{\circ} \widehat{\mathscr{F}}^{\mathscr{H}}$, then the product $\widehat{\mathscr{F}^{\mathscr{H}}} \circ \widehat{\mathscr{G}^{\mathscr{H}}} \in \widehat{\wp \mathscr{H}(m, n)}$. Proof. Let $\widehat{\mathscr{F}^{\mathscr{H}}} \circ \widehat{\mathscr{G}^{\mathscr{H}}}=\widehat{\mathscr{G}}^{\circ} \widehat{\mathscr{F}^{\mathscr{H}}}$. Then, we have

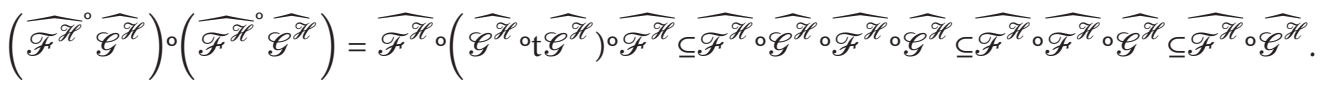

Therefore, $\widehat{\mathscr{F}^{\mathscr{H}}} \circ \widehat{\mathscr{G}^{\mathscr{H}}}$ is a HFSS of $S$. Also, we have

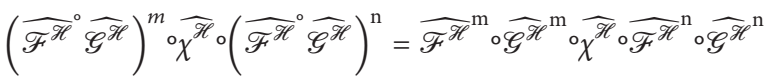
$\subseteq \widehat{\mathscr{F}}^{m} \circ \widehat{\mathscr{H}}^{\circ} \widehat{\mathscr{G}}^{\mathrm{H}} \mathrm{\textrm {n }} \widehat{\mathscr{F}}^{\mathscr{H}} \subseteq \widehat{\mathscr{F}}^{\mathrm{m}} \circ \widehat{\mathscr{G}}^{\mathscr{H}} \circ \widehat{\mathscr{F}}^{\mathscr{H}} \mathrm{n}$ $\widehat{\subseteq \mathscr{F}^{\mathscr{H}}} \circ \widehat{\mathscr{G}^{\mathscr{H}}} \circ \widehat{\mathscr{F}^{\mathscr{H}}} \subseteq \widehat{\mathscr{F}^{\mathscr{H}}} \circ \widehat{\mathscr{G}^{\mathscr{H}}}$,

as required.

Lemma 12. Let $\widehat{\mathscr{F}^{\mathscr{H}}}$ be a HFS of $S$. Then,

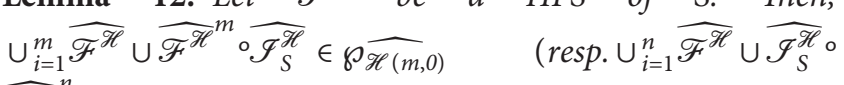
$\left.\widehat{\mathscr{F}}^{n} \in \widehat{\mathcal{H}_{(0, n)}}\right)$.

Proof. Straightforward.

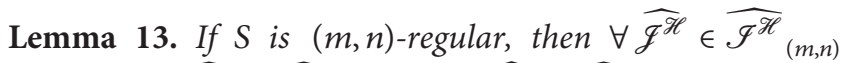
there exist $\widehat{\mathscr{F}}^{\mathscr{H}} \in{\widehat{\mathscr{I}^{\mathscr{H}}}}_{(m, 0)}$ and $\widehat{\mathscr{G}}^{\mathscr{H}} \in \widehat{\mathscr{I}}_{(0, n)}$ such that $\widehat{\mathscr{J}^{\mathscr{H}}}=\widehat{\mathscr{F}^{\mathscr{H}}} \circ \widehat{\mathscr{G}^{\mathscr{H}}}$.

Proof. Let $\widehat{\mathscr{J}^{\mathscr{H}}} \in{\widehat{\mathscr{I}^{\mathscr{H}}}}_{(m, n)}$. Then, ${\widehat{\mathscr{J}^{\mathscr{H}}}}^{m} \circ \widehat{\mathscr{I}}_{\mathrm{S}}^{\mathscr{H}} \circ{\widehat{\mathcal{J}^{\mathscr{H}}}}^{\mathrm{n}} \subseteq \widehat{\mathscr{J}}^{\mathscr{H}}$. As $S$ is $(m, n)$-regular, $\quad \widehat{J}^{\mathscr{H}} \subseteq \widehat{\mathscr{J}}^{m} \circ \widehat{\mathscr{J}}_{\mathrm{S}}^{\mathscr{H}} \circ \widehat{\mathscr{J}}^{\mathscr{H}} \mathrm{n}$. Therefore, $\widehat{\mathscr{J}^{\mathscr{H}}}={\widehat{\mathscr{J}^{\mathscr{H}}}}^{m} \circ \widehat{\mathscr{I}}_{\mathrm{S}}^{\mathscr{H}} \circ \widehat{\mathscr{J}}^{\mathscr{H}}$. Let $\widehat{\mathscr{F}^{\mathscr{H}}}=\cup_{i=1}^{m}{\widehat{\mathscr{J}^{\mathscr{H}}}}^{i} \cup{\widehat{J^{\mathscr{H}}}}^{m} \circ \widehat{\mathscr{I}}_{\mathrm{S}}^{\mathscr{H}}$ and $\quad \widehat{\mathscr{G}^{\mathscr{H}}}=\cup_{i=1}^{n} \widehat{\mathscr{J}}^{i} \cup \widehat{\mathscr{I}}_{S}^{\mathscr{H}} \circ \widehat{\mathscr{J}}^{\mathscr{H}^{\mathrm{n}}}$. By Lemma 12, $\widehat{\mathscr{F}^{\mathscr{H}}} \in \widehat{\wp_{\mathscr{H}(m, 0)}}$ and $\widehat{\mathscr{G}^{\mathscr{H}}} \in \widehat{\wp_{\mathscr{H}(0, n)}}$. As $S$ is $(m, n)$-regular, $\widehat{\mathscr{F}^{\mathscr{H}}}=\cup_{i=1}^{m}{\widehat{\mathscr{J}^{\mathscr{H}}}}^{i} \cup{\widehat{\mathscr{J}^{\mathscr{H}}}}^{m} \circ \widehat{\mathscr{I}}_{\mathrm{S}}^{\mathscr{H}}=\widehat{\mathscr{J}}^{\mathscr{H}} \mathrm{m} \widehat{\mathscr{J}}_{\mathrm{S}}^{\mathscr{H}}$ $\widehat{\mathscr{G}^{\mathscr{H}}}=\cup_{i=1}^{n} \widehat{\mathscr{J}}^{i} \cup \widehat{\mathscr{I}}_{S}^{\mathscr{H}} \circ \widehat{\mathscr{J}}^{\mathscr{H}} \mathrm{n}=\widehat{\mathscr{I}}_{\mathrm{S}}^{\mathscr{H}} \circ \widehat{\mathscr{J}}^{\mathscr{H}} \mathrm{n}$. Thus,

$$
\begin{aligned}
& \widehat{\mathscr{F}}^{\circ} \widehat{\mathscr{G}}^{\mathscr{H}}=\widehat{\mathscr{J}}^{m} \widehat{\mathscr{I}}_{\mathrm{S}}^{\mathscr{H}}{\widehat{\mathscr{I}_{\mathrm{S}}^{\mathscr{H}}}}^{\circ} \widehat{\mathscr{J}}^{\mathscr{H}} \subseteq \widehat{\mathscr{J}}^{\mathrm{H}} \mathrm{m} \cdot \widehat{\mathscr{I}_{\mathrm{S}}^{\mathscr{H}}} \circ \widehat{\mathscr{J}}^{\mathrm{H}} \mathrm{n}=\widehat{\mathscr{J}}^{\mathscr{H}}
\end{aligned}
$$

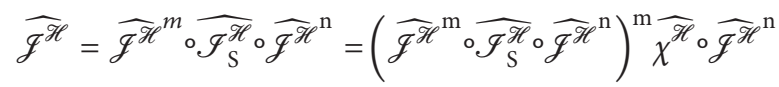

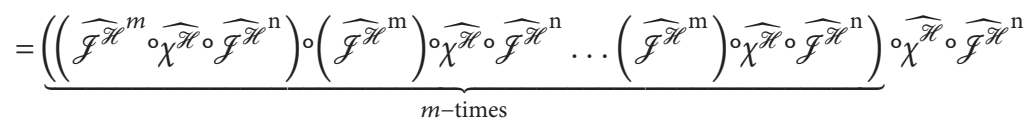

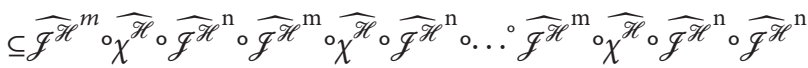

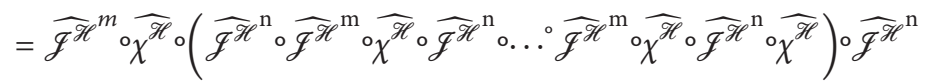

$$
\begin{aligned}
& \subseteq \widehat{\mathscr{J}}^{m}{ }^{m} \chi^{\mathscr{H}} \circ \chi^{\mathscr{H}} \circ \mathscr{H}^{\mathrm{n}}=\widehat{\mathscr{F}^{\mathscr{H}}} \circ \widehat{\mathscr{G}^{\mathscr{H}}},
\end{aligned}
$$

as required.

Lemma 14. If $\underline{S}$ is $(m, n)$-regular, then $\forall \widehat{\mathscr{F}^{\mathscr{H}}} \in \wp_{\mathscr{\mathscr { H }}(m, 0)}$, and for each HFS $\mathscr{G}^{\mathscr{H}}$ of $S, \mathscr{F}^{\mathscr{H}} \circ \mathscr{G}^{\mathscr{H}} \in \widehat{\wp} \widehat{\mathscr{H}(m, n)}$. 
Proof. Let $\widehat{\mathscr{F}^{\mathscr{H}}} \in \widehat{\wp \mathscr{H}(m, 0)}$ and $\widehat{\mathscr{G}^{\mathscr{H}}}$ be the HFS of $S$. We have

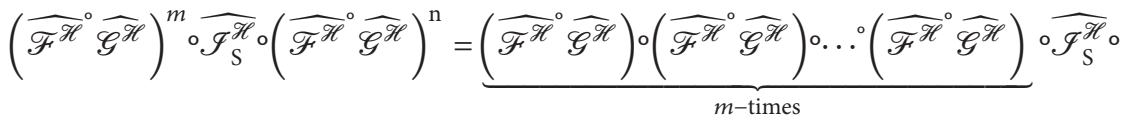

$$
\begin{aligned}
& \underbrace{\left(\widehat{\mathscr{F}}^{\circ} \widehat{\mathscr{G}}^{\mathscr{H}}\right) \circ\left(\widehat{\mathscr{F}}^{\circ} \widehat{\mathscr{G}}^{\mathscr{H}}\right)^{\circ} \cdots\left(\widehat{\mathscr{F}}^{\circ} \widehat{\mathscr{G}}^{\mathscr{H}}\right)}_{n \text {-times }}
\end{aligned}
$$

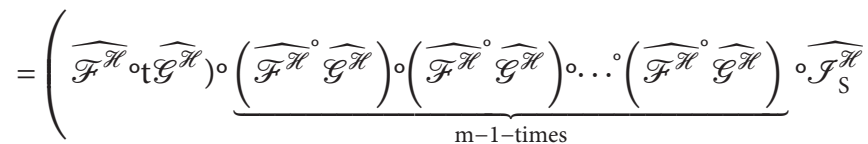

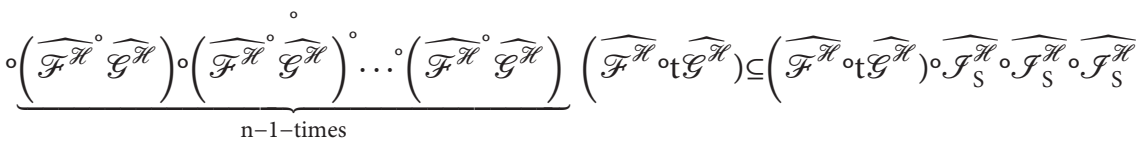

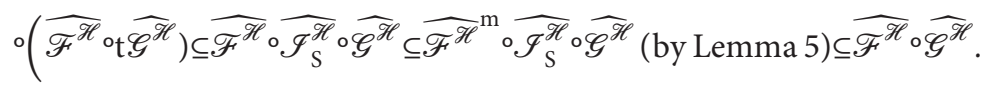

Therefore, $\widehat{\mathscr{F}^{\mathscr{H}}} \circ \widehat{\mathscr{G}^{\mathscr{H}}} \in \widehat{\wp} \widehat{\mathscr{H}(\mathrm{m}, \mathrm{n})}$.

By Lemmas 13 and 14, we have the following.

Corollary 1. If $S$ is $(m, n)$-regular, then $\widehat{\mathscr{J}^{\mathscr{H}}} \in \widehat{\wp_{\mathscr{H}(m, n)}} \Leftrightarrow$ there exist $\widehat{\mathscr{F}^{\mathscr{H}}} \in \widehat{\wp \mathscr{H}(m, 0)}$ and $\widehat{\mathscr{G}^{\mathscr{H}}} \in \widehat{\wp_{\mathscr{H}(0, n)}}$ such that $\widehat{\mathscr{J}}^{\mathscr{H}}=\widehat{\mathscr{F}}^{\circ} \widehat{\mathscr{G}}^{\mathscr{H}}$.

\section{Conclusion}

The principal objective of this paper is to establish the notions of the hesitant fuzzy - ideal, hesitant fuzzy $(m, 0)$-ideal, and hesitant fuzzy $(0, n)$-ideal and to improve the understanding of various semigroup classes through the use of these notions. In particular, if we take $m=1=n$ in the hesitant fuzzy $(m, n)$-ideal, hesitant fuzzy $(m, 0)$-ideal, and hesitant fuzzy $(0, n)$-ideal, then we get the hesitant fuzzy bi-ideal, hesitant fuzzy right ideal, and hesitant fuzzy left ideal. The concepts presented in this paper are therefore more general. Furthermore, if we put $m=1=n$ in the results of this paper, then most of the results of the paper [?] are deduced as corollaries which are the key application of the findings of this paper and a proof of the genuineness of the notions presented in this paper.

\section{Data Availability}

No data were used to support this study.

\section{Conflicts of Interest}

The authors declare that they have no conflicts of interest.

\section{Authors' Contributions}

All authors contributed equally to the manuscript.

\section{Acknowledgments}

This work was supported by the Taif University Researchers Supporting Project (TURSP-2020/246), Taif University, Taif, Saudi Arabia.

\section{References}

[1] V. Torra, "Hesitant fuzzy sets," International Journal of Intelligent Systems, vol. 25, pp. 529-539, 2010.

[2] V. Torra and Y. Narukawa, "On hesitant fuzzy sets and decision," in Proceedings of the 18th IEEE International Conference on Fuzzy Systems, pp. 1378-1382, Jeju Island, Korea, 2009.

[3] Y. B. Jun, M. A. Ozturk, and G. Muhiuddin, "A novel generalization of fuzzy subsemigroups," Annals of Fuzzy Mathematics and Informatics, vol. 14, no. 4, pp. 359-370, 2017.

[4] Y. B. Jun, S. Z. Song, and G. Muhiuddin, "Concave soft sets, critical soft points, and union-soft ideals of ordered semigroups," The Scientific World Journal, vol. 2014, Article ID 467968, 11 pages, 2014.

[5] G. Muhiuddin, N. Rehman, and Y. B. Jun, "A generalization of $(\epsilon, \in \vee q)$-fuzzy ideals in ternary semigroups," Annals of Communications in Mathematics, vol. 2, no. 2, pp. 73-83, 2019.

[6] G. Muhiuddin, "Neutrosophic subsemigroups," Annals of Communication in Mathematics, vol. 1, no. 1, pp. 1-10, 2018.

[7] G. Muhiuddin, A. Mahboob, and A. Mahboob, "Int-soft ideals over the soft sets in ordered semigroups," AIMS Mathematics, vol. 5, no. 3, pp. 2412-2423, 2020.

[8] G. Muhiuddin, "Cubic interior ideals in semigroups," Applications and Applied Mathematics, vol. 14, no. 1, pp. 463474, 2019.

[9] G. Muhiuddin, A. Mahboob, and N. Mohammad Khan, "A new type of fuzzy semiprime subsets in ordered semigroups," 
Journal of Intelligent \& Fuzzy Systems, vol. 37, no. 3, pp. 4195-4204, 2019.

[10] G. Muhiuddin, D. Al-Kadi, and M. Balamurugan, "Antiintuitionistic fuzzy soft a-ideals applied to BCI-algebras," Axioms, vol. 9, no. 3, p. 79, 2020.

[11] G. Muhiuddin, D. Al-Kadi, A. Mahboob, and K. P. Shum, "New types of bipolar fuzzy ideals of BCK-algebras," International Journal of Analysis and Applications, vol. 18, no. 5, pp. 859-875, 2020.

[12] G. Muhiuddin and K. P. Shum, "New types of $(\alpha, \beta)$ - fuzzy subalgebras of BCK/BCI-algebras," International Journal of Mathematics and Computer Science, vol. 14, no. 2, pp. 449464, 2019.

[13] G. Muhiuddin, A. Al-Kenani, E. Roh, and Y. Jun, "Implicative neutrosophic quadruple BCK-algebras and ideals," Symmetry, vol. 11, no. 2, p. 277, 2019.

[14] R. M. Rodriguez, L. Martinez, and F. Herrera, "Hesitant fuzzy linguistic term sets for decision making," IEEE Transactions on Fuzzy Systems, vol. 20, no. 1, pp. 109-119, 2012.

[15] G. Wei, "Hesitant fuzzy prioritized operators and their application to multiple attribute decision making," KnowledgeBased Systems, vol. 31, pp. 176-182, 2012.

[16] M. Xia and Z. Xu, "Hesitant fuzzy information aggregation in decision making," International Journal of Approximate Reasoning, vol. 52, no. 3, pp. 395-407, 2011.

[17] M. Xia, Z. Xu, and N. Chen, "Some hesitant fuzzy aggregation operators with their application in group decision making," Group Decision and Negotiation, vol. 22, no. 2, pp. 259-279, 2013.

[18] Z. Xu and M. Xia, "Distance and similarity measures for hesitant fuzzy sets," Information Sciences, vol. 181, no. 11, pp. 2128-2138, 2011.

[19] B. Zhu, Z. Xu, and M. Xia, "Hesitant fuzzy geometric Bonferroni means," Information Sciences, vol. 205, pp. 72-85, 2012.

[20] Y. B. Jun and S.-Z. Song, "Hesitant fuzzy set theory applied to filters in MTL-algebras," Honam Mathematical Journal, vol. 36, no. 4, pp. 813-830, 2014.

[21] Y. B. Jun and S.-Z. Song, "Hesitant fuzzy prefilters and filters of EQ-algebras," Applied Mathematical Sciences, vol. 9, pp. 515-532, 2015.

[22] Y. B. Jun, S. S. Ahn, and G. Muhiuddin, "Hesitant fuzzy soft subalgebras and ideals in BCK/BCI-algebras," The Scientific World Journal, vol. 2014, p. 7, Article ID 763929, 2014.

[23] Y. B. Jun and S. S. Ahn, "Hesitant fuzzy set theory applied to BCK/BCI-algerbas," Journal of Computational Analysis and Applications, vol. 20, no. 4, pp. 635-646, 2016.

[24] Y. B. Jun, K. J. Lee, and S.-Z. Song, "Hesitant fuzzy bi-ideals in semigroups," Communications of the Korean Mathematical Society, vol. 30, no. 3, pp. 143-154, 2015.

[25] Y. B. Jun, S.-Z. Song, and G. Muhiuddin, "Hesitant fuzzy semigroups with a frontier," Journal of Intelligent \& Fuzzy Systems, vol. 30, no. 3, pp. 1613-1618, 2016.

[26] G. Muhiuddin and S. Aldhafeeri, "Join hesitant fuzzy filters of residuated lattices," Italian Journal of Pure and Applied Mathematics, vol. 43, pp. 100-114, 2020.

[27] G. Muhiuddin, A. M. Alanazi, M. E. A. Elnair, and K. P. Shum, "Inf-hesitant fuzzy subalgebras and ideals in BCK/BCI-algebras," European Journal of Pure and Applied Mathematics, vol. 13, no. 1, pp. 9-18, 2020.

[28] G. Muhiuddin, A. Mahboob, and M. Balamurugan, "Hesitant anti-intuitionistic fuzzy soft commutative ideals of BCK-algebras," Annals of Communications in Mathematics, vol. 3, no. 2, pp. 158-170, 2020.
[29] G. Muhiuddin and B. Young, "Sup-hesitant fuzzy subalgebras and its translations and extensions," Annals of Communications in Mathematics, vol. 2, no. 1, pp. 48-56, 2019.

[30] A. F. Talee, M. Y. Abbasi, G. Muhiuddin, and S. A. Khan, "Hesitant fuzzy sets approach to ideal theory in ordered Gamma-semigroups," Italian Journal of Pure and Applied Mathematics, vol. 43, pp. 73-85, 2020.

[31] G. Muhiuddin and A. M. Al-roqi, "Regular hesitant fuzzy filters and MV -hesitant fuzzy filters of residuated lattices," Journal of Computational Analysis and Applications, vol. 24, no. 6, pp. 1133-1144, 2018.

[32] G. Muhiuddin and S. Aldhafeeri, "Subalgebras and ideals in BCK/BCI-algebras based on uni-hesitant fuzzy set theory," European Journal of Pure and Applied Mathematics, vol. 11, no. 2, pp. 417-430, 2018.

[33] G. Muhiuddin, E. H. Roh, S. Sun, and Y. B. Jun, "Hesitant fuzzy filters in lattice implication algebras," Journal of Computational Analysis and Applications, vol. 22, no. 6, pp. 1105-1113, 2017.

[34] G. Muhiuddin, H. S. Kim, S. Z. Song, and Y. B. Jun, "Hesitant fuzzy translations and extensions of subalgebras and ideals in BCK/BCI-algebras," Journal of Intelligent \& Fuzzy Systems, vol. 32, no. 1, pp. 43-48, 2017.

[35] G. Muhiuddin, "Hesitant fuzzy filters and hesitant fuzzy $G$-filters in residuated lattices," Journal of Computational Analysis and Applications, vol. 20, no. 2, pp. 394-404, 2016.

[36] S. Lajos, "Generalized ideals in semigroups," Acta Scientiarum Mathematicarum, vol. 22, pp. 217-222, 1961.

[37] M. Akram, N. Yaqoob, and M. Khan, "On (m,n)-ideals in LAsemigroups," Applied Mathematical Sciences, vol. 7, no. 44, pp. 2187-2191, 2013.

[38] L. Bussaban and T. Changphas, "On $(m, n)$-ideals on $(m, n)$-regular ordered semigroups," Songklanakarin Journal of Science and Technology, vol. 38, no. 2, pp. 199-206, 2016.

[39] T. Changphas, "On 0-minimal $(m, n)$-ideals in an ordered semigroup," International Journal of Pure and Applied Mathematics, vol. 89, no. 1, pp. 71-78, 2013.

[40] R. Tilidetzke, "A characterization of 0 -minimal $\$(m, n) \$-$ ideals," Czechoslovak Mathematical Journal, vol. 31, no. 1, pp. 48-52, 1981.

[41] N. Yaqoob and R. Chinram, "On prime $(m, n)$ bi-ideals and rough prime $\mathrm{b}(m, n) \mathrm{i}$-ideals in semigroups," Far East Journal of Mathematical Sciences, vol. 62, no. 2, pp. 145-159, 2012.

[42] A. Mahboob, N. M. Khan, and B. Davvaz, "Structural properties for $\$(m, n) \$$-quasi-hyperideals in ordered semihypergroups," Tbilisi Mathematical Journal, vol. 11, no. 4, pp. 145-163, 2018.

[43] A. Mahboob, N. M. Khan, and B. Davvaz, “( $m, n)$-hyperideals in Ordered Semihypergroups," Categories and General Algebraic Structures with Application, vol. 12, no. 1, pp. 43-67, 2020.

[44] D. N. Krgovic, "On $(m, n)$-regular semigroups," Publications De L'institut Mathematique, vol. 18, no. 32, pp. 107-110, 1975. 